\title{
Effect of time constraints on the conceptual representation of metaphor processing
}

\author{
Pei Liu ${ }^{1}$, Louise Connell ${ }^{1}$ and Dermot Lynott ${ }^{1,2}$ \\ ${ }^{1}$ Department of Psychology, Lancaster University \\ ${ }^{2}$ Department of Psychology, Maynooth University
}

\begin{abstract}
Author Note
We have no conflicts of interest to declare. Correspondence concerning this article should be addressed to Louise Connell (1.connell@1ancaster.ac.uk) or Dermot Lynott (dermot.lynott@,mu.ie)
\end{abstract}

Word count: I2609 


\begin{abstract}
What shapes the conceptual representation during metaphor processing? In this paper, we investigate this question by studying the roles of both embodied simulation and linguistic distributional patterns. Researchers have propose that the linguistic component is shallow and speedy, ideal as a shortcut to construct crude representations and conserve valuable cognitive resources. Thus, during metaphor processing, people should rely on the linguistic component more if the goal of processing is shallow and the time available is limited. Here, we present two pre-registered experiments which aim to evaluate this hypothesis. The results supported the role of simulation in metaphor processing, but not the linguistic shortcut hypotheses: the effect of linguistic distributional frequency increased as people had more time to process the metaphors, and as they engaged in deep processing. Furthermore during shallow processing, the processing was easier when the embodied and linguistic components support each other. These findings indicate a complex interaction between the embodied and linguistic components during metaphor processing.
\end{abstract}

Keywords: metaphor processing, embodied simulation, linguistic distributional pattern, linguistic shortcut hypothesis

Word count: 12609 words 
Effect of time constraints on the conceptual representation of metaphor processing

Metaphor is a crucial aspect of human language and cognition. A metaphoric expression applies to words and phrases where its meaning departs from the literal meaning of the expression. For instance, Student can be bright uses the word bright in a non-literal sense (i.e., "clever or intelligent"), while its literal meaning of "emitting much light" does not apply to the concept of student. Hence, it is not immediately apparent how people achieve comprehension in such a case. In this paper, we will consider the embodied and the linguistic components of conceptual representation, that is language processing relies on coarse-grained linguistic distributional information as well as fine-grained embodied simulation (e.g., Barsalou, Santos, Simmons, \& Wilson, 2008; Connell \& Lynott, 2014; Louwerse \& Jeuniaux, 2008). The linguistic distributional information describes patterns of how linguistic symbols (words or phrases) co-occur across language. As it is to do with linguistic symbols, the distributional patterns do not have a direct and causal link with the meaning. We will argue that the distributional patterns are utilised in metaphor processing as well as the embodied component, and test whether the linguistic distributional information can perform as a heuristic for metaphor processing, particularly under time pressure.

\section{Grounded Views of Language Processing}

Research on conceptual representations suggests that at least two components are employed for semantic representation when people process language (Barsalou et al., 2008; Connell \& Lynott, 2014; Louwerse \& Jeuniaux, 2008; Lynott \& Connell, 2010; Vigliocco, Meteyard, Andrews, \& Kousta, 2009). On one hand, representations are formed concerning the statistical, distributional pattern of how words co-occur across contexts. For example, the words bright and student occur 
together more often in language than do shining and student. Such distributional frequency patterns are able to explain to a great extent many aspects of language processing, such as priming and reading times (Vigliocco et al., 2009), language acquisition from early infancy (Aslin, Saffran, \& Newport, 1998; Monaghan, Chang, Welbourne, \& Brysbaert, 2017; Saffran, Johnson, Aslin, \& Newport, 1999), and even to some extent distinguishing words that relate to different perceptual modalities (Louwerse \& Connell, 2011).

On the other hand, past experiences of perceptual, motor, affective, introspective and other features are also partially reactivated during language processing (Allport, 1985; Barsalou, 1999; Glenberg \& Gallese, 2012; Niedenthal, 2007). Such reactivation of experiences, which is called simulation, forms the embodied component of a conceptual representation. Evidence for embodied simulation includes shared activation between brain areas involved in perceptual or motor experience and their equivalents in language comprehension. For example, reading sound-related words like thunder activates the auditory association cortex, and their processing is selectively impaired in patients with atrophy of the auditory association cortex (Bonner \& Grossman, 2012; Boulenger et al., 2008; R. F. Goldberg, Perfetti, \& Schneider, 2006). Together, the linguistic and embodied components can explain language processing better than either alone (Andrews, Vigliocco, \& Vinson, 2009; Johns \& Jones, 2012; Louwerse, 2011).

In particular, researchers have suggested that the linguistic component can provide a shortcut for superficial language processing (Barsalou et al., 2008; Connell \& Lynott, 2014; Louwerse \& Jeuniaux, 2008). During language processing, the activation of linguistic distributional patterns is easy and requires little effort, so it reaches the peak of its activation before embodied simulation. Furthermore, the co-occurrence of words in language often reflects the associations of objects, events, and ideas in the real world. The fact that bright appears with sun frequently in close 
proximity reflects the reality that the sun is usually bright. Therefore, the linguistic component is sufficient to generate a good-enough approximation (Ferreira, Bailey, \& Ferraro, 2002), especially when detailed semantic representation is not required (Connell \& Lynott, 2013, 2014; Louwerse, 2011; Louwerse \& Connell, 2011). In contrast, embodied simulation, though also activated immediately in language processing, is slower and more costly. Therefore, it is reasonable that people would rely on information from the linguistic component for a guide to whether it is worth expending effort on costly embodied simulation (Connell \& Lynott, 2013).

To test this proposal, Connell and Lynott (2013) asked participants to perform two tasks with novel noun-noun compounds (e.g., cactus beetle): a relatively shallow processing task where participants makes a yes/no judgement concerning whether the compounds made sense, or a deeper processing task where they decided if they could generate specific interpretations for them (and then provided the interpretation). The results showed that, when the linguistic component indicated that the processing was likely to fail (e.g., cactus and beetle, which rarely co-occur), people were more likely to reject the compound and rejected it more quickly, abandoning it before any more cognitive effort was expended. On the other hand, when the linguistic component indicates that future processing is likely to succeed (e.g., army and decision, which frequently co-occur), people's response strategy would be based on the requirement of the task. In the shallow sensibility judgment task, linguistic distributional frequency heavily affected the response decision and speed to accept a compound; whereas in the deep interpretation generation task, linguistic distributional frequency did not affect the speed to accept a compound. In other words, while rejecting a noun-noun compound can simply rely on linguistic shortcut to quickly flag up unsuccessful processing, accepting a compound as interpretable may require more detailed simulation which cannot be captured by the linguistic distributional frequency. 
The cognitive triage mechanism, though proposed for literal language processing, can equally be applied metaphor processing. Liu, Connell, and Lynott (2017) tested whether the mechanism worked in metaphor processing with a similar design as Connell and Lynott (2013). Going further than that study, they also introduced a measure of the effort to simulate a concept (Liu, Connell, \& Lynott, 2018). In this metaphor processing study, participants read metaphoric sentences such as Students can be bright or Supporters can be lukewarm, and performed either a yes/no sensibility judgement task or an interpretation generation task. The results of the metaphor processing study showed that, while ease of simulation affected the speed of processing in both tasks, linguistic distributional frequency only affected the speed of processing in the relatively shallow, sensibility judgment task. That is, the co-occurrence of the words students and bright predicted how quickly people judged the metaphor Students can be bright to be sensible, but did not predict how quickly they could generate a specific interpretation for the metaphor (e.g., "clever students"). These results, on the face value, seemed to support the triage mechanism and the linguistic shortcut hypothesis. However, contrary to the idea that people would rely on linguistic distributional frequency more in shallow processing, linguistic distributional frequency did not have a reliably larger effect on response time in the shallow sensibility judgment task than the deep interpretation generation task. Furthermore, linguistic distributional frequency had a greater effect on the rate of acceptance in the deep interpretation generation task than the in shallow sensibility judgment task. This pattern of results seems to suggest the opposite of the linguistic heuristic hypothesis: that is, people relied on the linguistic component more heavily when they were required to process a metaphor deeply and generate a detailed mental representation.

One possible explanation for the mixed results could be that the use of the linguistic shortcut needs to be incentivised. That is to say, in order for people to 
rely on linguistic distributional patterns rather than detailed simulated information, they need to be given conditions that induces a speed-accuracy tradeoff. The advantage of the linguistic shortcut is that it conserves limited cognitive capacity and time compared to the more costly and time-consuming, full-fledged simulation. Therefore, people should be more reliant on the linguistic shortcut if the resources (of time or processing capacity) are limited.

\section{Present Study}

In the present study, we will put people under different levels of time constraints while they perform the same metaphor processing tasks as the previous study (Liu et al., 2017). For a sentence such as Supply can be bright, the linguistic distributional pattern should first suggest that the constituent words supply and bright often co-occur, thus the processing likely to succeed. In such a case, people could be encouraged to take the linguistic shortcut if they have limited time to make a response. Therefore, they may either accept the metaphor straight away based on the high distributional frequency even though the embodied component has not produced a coherent simulation yet, or they could allow the embodied component to engage in deeper and more costly simulation. In contrast, for a sentence such as Illness can be bright, the linguistic component should immediately suggest that the two constituent words rarely co-occur. Based on this information, people may quickly reject the metaphor to avoid time running out or they may only allow the embodied component to process the metaphor in a very small degree. In both cases, the shorter the time is available, the more people should rely on the linguistic component, whereas if people have more time, they can be free to engage in time-consuming simulations.

Additionally, we expect to replicate the effects of linguistic distributional frequency and ease of simulation from the previous processing study. That is, we 
expect them to affect the judgement and speed of processing independently. People should be more likely and faster to accept a metaphor as sensible or interpretable when ease of simulation is high, and less likely and slower to reject it when ease of simulation is low. Linguistic distributional frequency should further predict a unique portion of response decision and speed, which should be in the same direction as ease of simulation. Task will further moderate the effect of both components, such that ease of simulation will have a larger effect during deeper processing than shallow processing, while vice versa for linguistic distributional frequency.

The main studies with the hypotheses and the method were preregistered on AsPredicted.org (http://aspredicted.org/zv3y2.pdf) and conducted as the pre-registration unless otherwise specified and justified. The pilot study, whose aim was to determine elements of design of the main studies, was not pre-registered due to its exploratory nature, but is reported fully in this manuscript. We report here in all three experiments how we determined our sample size, all data exclusions, all manipulations, and all measures in the study.

\section{Pilot Study}

A pilot study was conducted to determine the time constraints suitable for the experiments. The constraints were selected based on the results from the previous metaphor processing study (Liu et al., 2017). The short constraints were set so that about $50 \%$ of the trials per task were responded to within the constraint. The threshold was set to give participants the pressure to respond quickly but not so much that the task became impossible. Thus, the short constraints turned out to be 1s for the sensibility judgment task and 2s for the interpretation generation task. The long constraints were set at the point where the distribution of RT would naturally end if assumed symmetric. This criterion was to ensure that participants could naturally respond to most of the trials without pressure. Thus, the long 
constraints turned out to be 3s for the sensibility judgement task and $8 \mathrm{~s}$ for the interpretation generation task. Consequently, the medium constraints were set to be the middle point of the two, that was $2 \mathrm{~s}$ for the sensibility judgement task and $5 \mathrm{~s}$ for the interpretation generation task. In the pilot study, we tested if the short constraint was too difficult for participants. The task would be judged as too difficult if people responded "yes" for less than $30 \%$ of the trials for the sensibility judgement task and less than $40 \%$ for the interpretation generation task. This difficulty threshold was also determined based on the previous study in which the acceptance rate was $45.51 \%$ for the sensibility judgement task and $61.91 \%$ for the interpretation generation task. That is to say, we expected the task to be more difficult in the current study because of the time constraint. Therefore, the threshold of acceptance rate was lowered in the present study so that people were allowed to fail to process the majority of the metaphors, but not so low as to suggest that participants would fail completely.

\section{Participants}

Sixteen native speakers of English from the Department of Psychology, Lancaster University were tested for the pilot study (age: $M=26.26$ years, $S D=$ 4.10 years; male: six; left-handed: one), eight for the sensibility judgement task and eight for the interpretation generation task.

\section{Material}

The material contained 452 metaphoric sentences from the ease-of-simulation norms (see also Liu et al., 2017, 2018). Table 1 contains examples of the materials, while the full list is in the Supplementary Material. All sentences took the form "Noun can be adjective" (e.g., Students can be bright), composed of 113 uni-modal perceptual adjectives selected from the modality exclusivity norms of Lynott and 
Connell (2009) and Dantzig, Cowell, Zeelenberg, and Pecher (2011) (e.g., bright), each paired with four nouns so that the adjectives could elicit metaphoric meanings. By pairing each adjective with four nouns, the metaphors varied on the following two variables:

Ease of simulation (EoS). EoS measured indirectly the effort to successfully simulate the concept in a metaphoric sentence. For each sentence, EoS ranged from easy to difficult $(M=0.00, S D=1.00)$, which was obtained through a novel norming study by Liu et al. (2018) where people rated on the metaphors based on three criteria: sensibility (How much sense does the sentence make if you read it in text or heard it in conversation?), usability (How easy it would be for you to use the sentence in writing or in conversation?), and imaginability (How easy it is for you to imagine the concept described in the sentence?). One principle component was extracted from the ratings, the factor scores of which was used as a continuous measure of how easy it is to simulate the concept. This EoS measure managed to explain $91 \%$ of the original variance of the ratings and outperformed all the separate ratings in predicting the response time of a sensibility judgement task (Liu et al., 2018). Therefore, EoS could be argued to measure the underlying mechanism of comprehension, instead of the noise related to specific linguistic tasks, which was assumed to be simulation (Zwaan, 2004).

\section{Linguistic distributional frequency (LDF). LDF measured the} co-occurrence frequency of the constituent words in a sentence. For each sentence, LDF ranged from low to high $(M=2.95, S D=0.97)$, and was calculated as the log-transformed sum of the bi- to five-gram frequencies of the metaphor's constituent words in the Google Web1T Corpus (Brants \& Franz, 2006). To take the metaphor Students can be bright as an example, the LDF was the sum of the frequencies of "student ... bright" and "bright ... student" with zero, one, two, and three intervening words. It was then log transformed as $\log _{10}(\mathrm{LDF}+41)$ where 
Table 1

Sample metaphors, and their scores for EoS

and $L D F$

\begin{tabular}{lcc}
\hline Metaphor & EoS & LDF \\
\hline Illness can be bright. & -1.32 & 2.95 \\
Supply can be bright. & -1.02 & 3.72 \\
Solutions can be bright. & 1.41 & 3.11 \\
Students can be bright. & 1.84 & 4.08 \\
Minutes can be lukewarm. & -1.49 & 3.31 \\
Scheme can be lukewarm. & -0.78 & 1.61 \\
Supporters can be lukewarm. & 0.69 & 2.69 \\
Reaction can be lukewarm. & 1.03 & 3.45 \\
\hline
\end{tabular}

41 is the lowest non-zero frequency in the corpus (Connell \& Lynott, 2013). Among the four metaphors created for each adjective, LDF varied independently from of EoS (see Table 1$)$. The two variables had a mild correlation $(r=.26$, sharing only $7 \%$ of common variance), which was handled later during the analysis.

These variables did not contribute to the analysis of the pilot study data, but were used to construct balanced stimulus lists. The sentences were split into four lists of 113 items each, where each adjective appeared only once per list, and the distribution of easy/difficult to simulate and high/low distributional frequency was approximately equal across lists $\left(\operatorname{EoS}: F_{(3,448)}=0.03, p=.99 ; \mathrm{LDF}: F_{(3,448)}=0.09\right.$, $p=.96)$. Each participant saw only one list. 


\section{Procedure}

Participants read the metaphoric sentences and performed either of the two tasks. They were randomly assigned to one of the tasks and judged whether or not the sentence made sense, or whether they could think of a meaning for the sentence. Each trial was conducted as in Figure 1 in the two tasks except at the final screen when people needed to make a judgement. The adjectives remained on the screen until participants responded or the time ran out. For the sensibility judgement task, participants were given 1s to respond; for the interpretation generation task, they were given 2 s to respond. If their response was "yes" (i.e. they judged the sentences as sensible or meaningful), they would press the comma key (,); if "no", they would press the full stop key (.). In the interpretation generation task, they were then asked to type down the meaning if they had responded "yes". If they did not manage to give a response within the time limit, a feedback saying " ****TOO SLOW***" was given for $350 \mathrm{~ms}$.

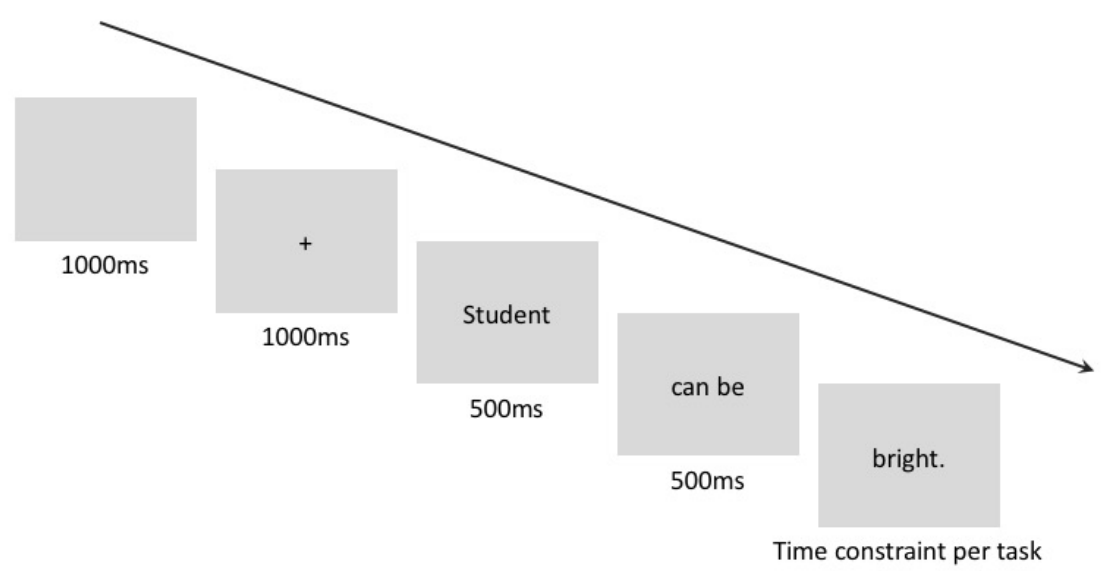

Figure 1. Trial structure of both sensibility judgement and interpretation generation. 


\section{Design and Analysis}

Response decisions and RT were recorded, but only response decisions were analysed. The "yes" response was categorised as accepted trials, the "no" response as rejected trials, and if the time elapsed without any responses, a missed trial.

\section{Results}

The sensibility judgement task had an average missing rate of $7.41 \%$ and an acceptance rate of $35.73 \%$. The interpretation generation task had an average missing rate of $3.32 \%$ and an acceptance rate of $49.45 \%$. Hence, we decided that the short time constraints for both tasks were acceptable and applied the time constraints to Experiments 1 and 2 .

\section{Experiment 1: Sensibility Judgement Task}

In this experiment, people performed sensibility judgements under three levels of time constraints (i.e., a short constraint of $1 \mathrm{~s}$, a medium constraint of $2 \mathrm{~s}$ and a long constraint of 3s). Since it only required a simple "yes" or "no" answer, the experiment encouraged people to engage in relatively shallow processing (Connell \& Lynott, 2013; Lynott \& Connell, 2010). We expected that while EoS would have an effect on the response decision and RT (i.e., high EoS, more and faster acceptance, less and slower rejection), LDF would also have an effect (i.e., high LDF, more and faster acceptance, less and slower rejection), especially under short time constraint. Furthermore, if people could simulate a metaphor extensively and in detail, the processing should end up being successful. Therefore, EoS should have a larger effect on the RT when the response was "yes" than "no. In contrast, because the linguistic shortcut could flag up potentially unsuccessful processing (i.e., those with 
low LDF), EoS should have a smaller effect and LDF should have a larger effect when the response was"no" than "yes".

\section{Participants}

Forty-eight students from Lancaster University participated in the study, all of whom were native speakers of English (age: $19.49(S D=2.91)$ years; male: seven; right-handed: 43). Participation took approximately 20 minutes in exchange for course credits. The sample size was determined based on a minimum acceptance rate of $35 \%$, which would provide a minimum number of data points per participant that was comparable to Liu et al. (2017); because this acceptance rate was achieved, it was not necessary to test any extra participants.

\section{Materials}

The 452 metaphoric sentence and item lists were the same as in the pilot study.

\section{Procedure}

The study was composed of three test blocks, one for each level of time constraint: short $(1 \mathrm{~s})$, medium $(2 \mathrm{~s})$, and long $(3 \mathrm{~s})$, to which the materials were randomly assigned. Participants were made aware that the time constraints might vary between blocks, although they did not know whether a given block would use a short, medium or long constraint. All items appeared under all time constraints across participants, and the order of blocks were counterbalanced. Before each block, participants were given ten practice trials in order to accustom them to the time-constrained response interval, during which their response was not recorded. The trial structure in the practice and test trials was identical to the pilot study. Participants were asked to respond "yes" if the sentence made sense and "no" if it 
did not make sense. Response decisions ("yes" or "no") were recorded for each trial, and response times were measured from the onset of the adjective until the keypress of the response decision.

\section{Design and Analysis}

This experiment had EoS and LDF as two continuous predictors, and three levels of time constraints (i.e., short, medium and long) were applied as within-subject categorical predictors. The time constraints were backward difference coded so that Contrast 1 was the difference of long minus medium constraints, and Contrast 2 is the difference of medium minus short constraints. The coding was not specified in the pre-registration, but it allowed us to spot the change of the effects of the two continuous predictors as time constraint tightened. Response decisions ("yes" or "no" responses) and response time (RT in millisecond) were the dependent variables. The response decisions were coded as 1 for "yes" response and 0 for "no" response.

The analyses were conducted according to the pre-registration. ${ }^{1}$ Response

\footnotetext{
${ }^{1}$ We used R (Version 3.5.0; R Core Team, 2017) and the R-packages abind (Version 1.4.5; Plate \& Heiberger, 2016), arm (Version 1.10.1; Gelman \& Su, 2016), BayesFactor (Version 0.9.12.4.2; Morey \& Rouder, 2015), bookdown (Version 0.7; Xie, 2016), broom (Version 0.4.4; Robinson, 2017), citr (Version 0.2.0; Aust, 2016), coda (Version 0.19.1; Plummer, Best, Cowles, \& Vines, 2006), data.table (Version 1.11.4; Dowle \& Srinivasan, 2017), ggplot2 (Version 2.2.1; Wickham, 2009), gridExtra (Version 2.3; Auguie, 2017), interplot (Version 0.1.5; Solt \& Hu, 2015), knitr (Version 1.20; Xie, 2015), lme4 (Version 1.1.17; Bates, Mächler, Bolker, \& Walker, 2015), lmerTest (Version 3.0.1; Kuznetsova, Brockhoff, \& Christensen, 2017), magrittr (Version 1.5; Bache \& Wickham, 2014), MASS (Version 7.3.50; Venables \& Ripley, 2002), Matrix (Version 1.2.14; Bates \& Maechler, 2017), MuMIn (Version 1.40.4; Bartoń, 2017), nlme (Version 3.1.137; Pinheiro, Bates, DebRoy, Sarkar, \& R Core Team, 2017), papaja (Version 0.1.0.9735; Aust \& Barth, 2017), psych (Version 1.8.4; Revelle, 2017), purrr (Version 0.2.5; Henry \& Wickham, 2017), and rcartocolor (Version
} 
decisions $^{2}$ were analysed using logistic mixed-effect regressions with centred EoS, centred LDF, levels of time constraints and their interactions as fixed predictors, and participant and item as crossed random-intercept factors. ${ }^{3}$ Furthermore (not pre-registered but consistent with our hypotheses), we also analysed the response decision per time constraint in order to model the effects of the predictors within each time constraint individually. $\mathrm{RT}^{4}$ were analysed using linear mixed-effect regressions with the same fixed predictor plus response decision, and the random-intercept factors as the mixed-effect logistic regressions. We also analysed the RT per response decision because we had different hypothesis for "yes" and "no" responses.

In these analyses, we also considered variables such as lexical frequency (sum of the log transformed frequencies of the component words) and sentence length (sum of the length of the component words) as additional predictors as pre-registered.

0.0.22; Nowosad, 2017) for all our analyses and the writing up of this manuscript.

2 The inclusion of participants as a random factor improved model fit above the empty model, $\chi^{2}(1)=394.00, p<.001$, as did the inclusion of items as a crossed random factor above the participants-only model, $\chi^{2}(1)=753.31, p<.001$.

3 Although it has been suggested that that mixed-effect models with random intercept and slope generalises better than a random-intercept-only model (Barr, Levy, Scheepers, \& Tily, 2013), we were not able to fit maximal models to our data because of several reasons. First, such a model (with two random predictors and three or four fixed predictors and their interactions) would require a huge amount of data for the parameter estimation which our study could not afford. Second, fitting such models is time consuming, often taking hours or days if performed in R, which makes tweaks and model comparisons impractical.

4 The inclusion of participants as a random factor improved model fit above the empty model, $\chi^{2}(1)=785.53, p<.001$. The inclusion of items as a crossed random factor did not improve model fit above the participants-only model $\chi^{2}(1)=0.00, p=1$. However, in order to keep the models consistent, both participants and items were included as crossed random factors in the linear models of RT as well as in the logistic models of response decision. 
However, the correlation between these variables and RT were near zero $(r<.10)$, so they were not included in the regression models (see Supplementary Material). Moreover in the analyses of RT, we found evidence of net suppression and problems with multicolinearity [J. Cohen, Cohen, West, and Aiken (2003); see Supplementary Materials]. This means that the shared error variance between LDF and EoS is effectively hiding the real relationship between the key variables and RT, such that the net effect of LDF was to enhance the effect of EoS by suppressing the latter's unhelpful error variance. Therefore, consistent with our pre-registration, EoS and LDF were centred and orthogonalised using principle components analysis with varimax rotation and Kaissar normalisation on a model by model basis. For clarity and space, we reported only results with orthogonalised variables for RT (for results with original variables, see Supplementary Material), and referred to these orthogonalised variables with their original labels (i.e., EoS instead of orthogonalised EoS, LDF instead of orthogonalised LDF). In the analysis of response decision, we did not find evidence of net suppression, so we did not orthogonalise the variables.

\section{Results and Discussion}

All participants had their mean response time within 3SD of the overall mean, so all were included in analysis. Three trials were excluded for motor error $(\mathrm{RT}<$ 200ms). Individual trials were excluded as outliers if the RT was more than 3SD from each participant's mean per response decision per level of time constraint. This exclusion was done differently from the pre-registration, in which we proposed to exclude outliers per response decision only, because we realised that this approach would have affected responses under short constraint and long constraint disproportionately. The percentage of outlying trials were: for "yes" responses, $0.15 \%$ under short constraint, $0.28 \%$ for medium constraint, and none for long constraint; for "no" responses, none for short constraint, $0.64 \%$ for medium 
constraint, $0.36 \%$ for long constraint. Consequently, 5261 trials were counted as valid trials and were used in analysis.

Response decisions. Overall, 2059 trials were accepted as sensible (acceptance rate: 39.14\%); 3202 trials were rejected as nonsensical (rejection rate: $60.86 \%$ ). For each level of time constraint, the acceptance rate was $39.23 \%$ for short constraint (656 among 1672 trials), 39.18\% for medium constraint (701 among 1789 trials), and 39.00\% for long constraint (702 among 1800 trials).

Results of the logistic mixed-effect regression are in Table 2. Only EoS had a significant and positive effect. It means that the easier the metaphor was normally regarded to simulate, the more likely it is to be judged as sensible (odds ratio $=$ 3.26). Furthermore, the effect of EoS changed between short time constraint and medium time constraint, being larger for medium than short. Besides, there was also a trend that the effect of EoS became slightly larger as LDF increased. When separated by the levels of time constraint (Table 3), EoS had an effect for all three levels of time constraint. Additionally, the slight trend of interaction between EoS and LDF appeared only for short time constraint but not for medium or long constraints.

Response time. The overall mean RT was $856 \mathrm{~ms}$ for "yes" responses $(S D=$ $312 \mathrm{~ms})$ and $838 \mathrm{~ms}$ for "no" responses $(S D=305 \mathrm{~ms})$. Separated by time constraint, the mean RT for "yes" responses was $687 \mathrm{~ms}(S D=156 \mathrm{~ms})$ for short constraint, $863 \mathrm{~ms}(S D=272 \mathrm{~ms})$ for medium constraint, and $1008 \mathrm{~ms}(S D=373 \mathrm{~ms})$ for long constraint; the mean RT for "no" response was $688 \mathrm{~ms}(S D=140 \mathrm{~ms})$ for short constraint, $839 \mathrm{~ms}(S D=251 \mathrm{~ms})$ for medium constraint, and $974 \mathrm{~ms}(S D=390 \mathrm{~ms})$ for long constraint.

In the omnibus model of RT (Table 4), we found that the time constraints did have a significant effect on RT. Participants responded more slowly as the time 
Table 2

Logistic mixed-effect regression of response decision in Experiment 1

\begin{tabular}{lrrr}
\hline & $b$ & $95 \%$ CI & $p$ \\
\hline Intercept & -0.64 & $-0.93--0.36$ & $<.001 * * *$ \\
Long - medium & -0.01 & $-0.18-0.16$ & .932 \\
Medium - short & -0.01 & $-0.18-0.16$ & .896 \\
LDF & 0.06 & $-0.03-0.16$ & .177 \\
EoS & 1.18 & $1.08-1.28$ & $<.001 * * *$ \\
Long - medium x LDF & -0.08 & $-0.27-0.1$ & .383 \\
Medium - short x LDF & -0.01 & $-0.19-0.18$ & .948 \\
Long - medium x EoS & -0.12 & $-0.31-0.07$ & .225 \\
Medium - short x EoS & 0.38 & $0.19-0.57$ & $<.001 * * *$ \\
LDF x EoS & 0.08 & $-0.01-0.17$ & $.084 \dagger$ \\
Long - medium x LDF x EoS & -0.03 & $-0.21-0.16$ & .776 \\
Medium - short x LDF x EoS & -0.04 & $-0.22-0.15$ & .714 \\
\hline
\end{tabular}

Note. $\dagger$ means $p<.10{ }^{*}$ means $p<.05$;

** means $p<.01 ; * * *$ means $p<.001$.

$b$ is non-standardised regression coefficient. 
Table 3

Logistic regression model of response decision per time constraint in Experiment 1

\begin{tabular}{llrrr}
\hline & & $b$ & $95 \%$ CI & $p$ \\
\hline \multirow{4}{*}{ Short } & Intercept & -0.62 & $-0.92--0.32$ & $<.001 * * *$ \\
& LDF & 0.09 & $-0.05-0.23$ & .197 \\
& EoS & 0.96 & $0.81-1.11$ & $<.001 * * *$ \\
& LDF x EoS & 0.11 & $-0.02-0.24$ & $.097 \dagger$ \\
\cline { 2 - 5 } Medium & Intercept & -0.64 & $-0.93--0.36$ & $<.001 * * *$ \\
& LDF & 0.08 & $-0.06-0.22$ & .266 \\
& EoS & 1.31 & $1.15-1.48$ & $<.001 * * *$ \\
& LDF x EoS & 0.07 & $-0.07-0.22$ & .319 \\
\cline { 2 - 5 } Long & Intercept & -0.70 & $-1.06--0.35$ & $<.001 * * *$ \\
& LDF & 0.01 & $-0.14-0.16$ & .859 \\
& EoS & 1.30 & $1.13-1.48$ & $<.001 * * *$ \\
& LDF x EoS & 0.06 & $-0.09-0.2$ & .457 \\
\hline
\end{tabular}

Note. $\dagger$ means $p<.10 ;{ }^{*}$ means $p<.05$;

** means $p<.01 ; * * *$ means $p<.001$.

$b$ is non-standardised regression coefficient. 
Table 4

Omnibus linear mixed-effect model of RT in Experiment 1

\begin{tabular}{|c|c|c|c|}
\hline & $b$ & $95 \% \mathrm{CI}$ & $p$ \\
\hline Intercept & 845.92 & $808.68-883.17$ & $<.001 * * *$ \\
\hline Long - medium & 129.20 & $106.25-152.16$ & $<.001 * * *$ \\
\hline Medium - short & 158.75 & $135.54-181.96$ & $<.001 * * *$ \\
\hline Response & 18.48 & $1.72-35.24$ & $.031 *$ \\
\hline $\mathrm{LDF}$ & -0.93 & $-11.76-9.9$ & .867 \\
\hline EoS & 14.80 & $3.49-26.11$ & $.010 * *$ \\
\hline Long - medium x Response & 55.87 & $17.35-94.39$ & $.004 * *$ \\
\hline Medium - short x Response & 36.72 & $-1.8-75.23$ & $.062 \dagger$ \\
\hline Long - medium $\mathrm{x}$ LDF & -0.55 & $-24.45-23.36$ & .964 \\
\hline Medium - short x LDF & 3.13 & $-21.12-27.38$ & .800 \\
\hline Response $\mathrm{x}$ LDF & -1.99 & $-18-14.03$ & .808 \\
\hline Long - medium x EoS & -7.11 & $-31.89-17.67$ & .574 \\
\hline Medium - short x EoS & 19.71 & $-5.3-44.71$ & .122 \\
\hline Response x EoS & -56.54 & $-72.8--40.29$ & $<.001 * * *$ \\
\hline LDF $x$ EoS & -13.28 & $-23.68--2.88$ & $.012 *$ \\
\hline Long - medium x Response x LDF & -18.30 & $-57.56-20.95$ & .361 \\
\hline Medium - short x Response x LDF & 7.21 & $-31.58-46.01$ & .715 \\
\hline Long - medium x Response x EoS & -71.38 & $-109.79--32.98$ & $<.001 * * *$ \\
\hline Medium - short x Response x EoS & -54.82 & $-93.23--16.41$ & $.005^{* *}$ \\
\hline Long - medium x LDF x EoS & -21.05 & $-43.61-1.52$ & $.068 \dagger$ \\
\hline Medium - short x LDF x EoS & 3.26 & $-19.91-26.43$ & .783 \\
\hline Response x LDF x EoS & 12.16 & $-3.2-27.53$ & .121 \\
\hline Long - medium x Response x LDF x EoS & 25.89 & $-10.54-62.33$ & .164 \\
\hline Medium - short x Response x LDF x EoS & -15.00 & $-50.93-20.92$ & .413 \\
\hline
\end{tabular}

Note. $\dagger$ means $p<.10{ }^{*}$ means $p<.05$;

** means $p<.01$; *** means $p<.001$. 
constraints got longer. EoS had a positive effect on "no" response when LDF was at its mean, meaning that the RT became longer as EoS increased. This effect was reversed for "yes" response (i.e., RT became shorter as EoS increased) and the effect on "yes" response was larger than that on "no" response. Furthermore, LDF moderated the effect of EoS on "no" response such that the effect of EoS became smaller (less positive) as LDF increased, which existed only for the long and medium constraints (reference levels of the contrast coding) and was smaller at medium than long constraints. 


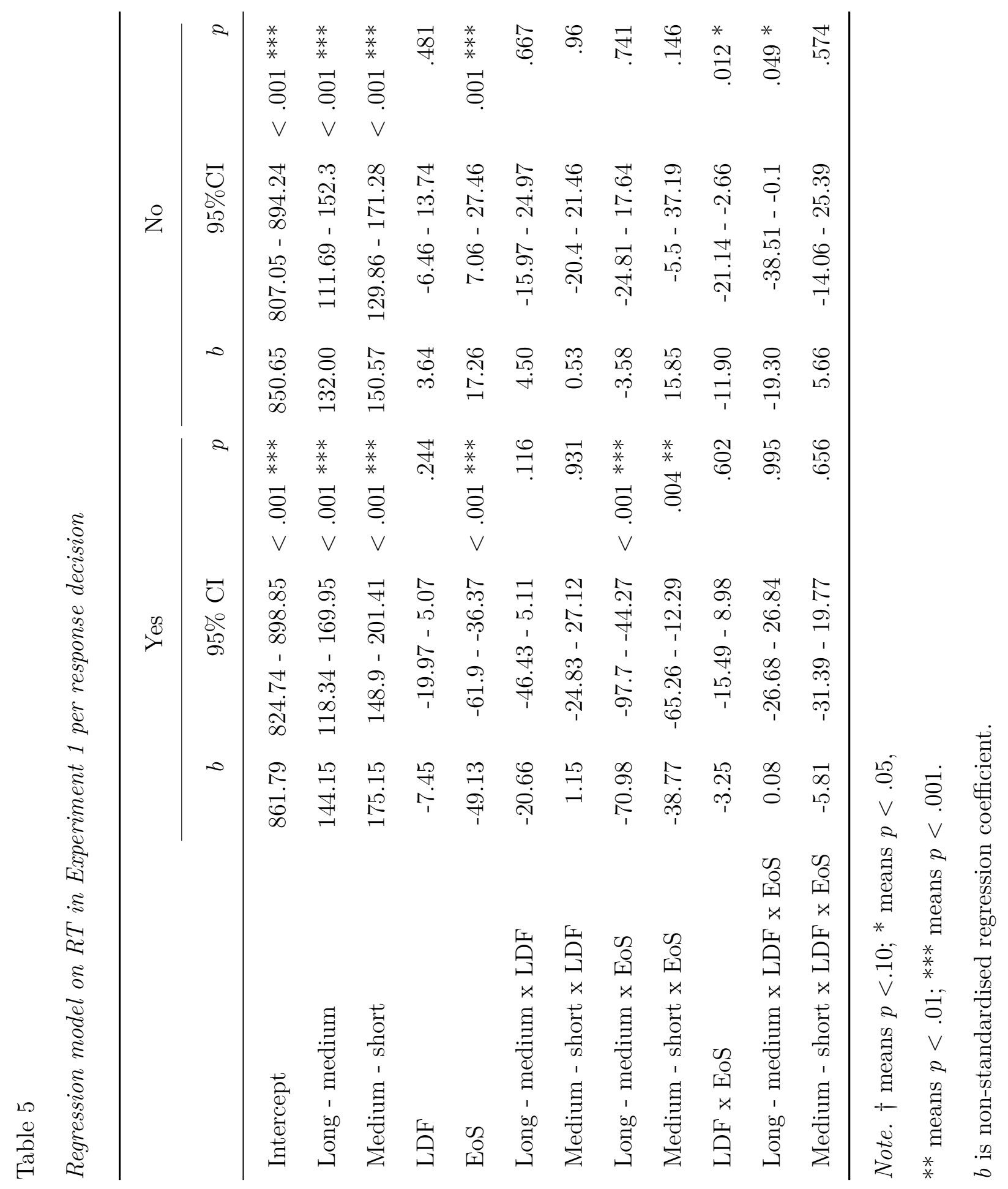


When separated by response decision (Table 5), EoS had a negative effect on RT for "yes" response during long and medium constraints. That is, the easier a metaphor was typically considered to simulate, the faster people accepted a metaphor as sensible. This effect was larger at the longer constraints than the short constraint (Table 6 and Figure 2). Analyses per time constraint also confirmed that EoS did not have a significant effect on "yes" RT at short time constraint, but only did during medium and long constraint (Table 11 and Figure 2). In contrast, LDF did not have a significant effect on "yes" RT. 


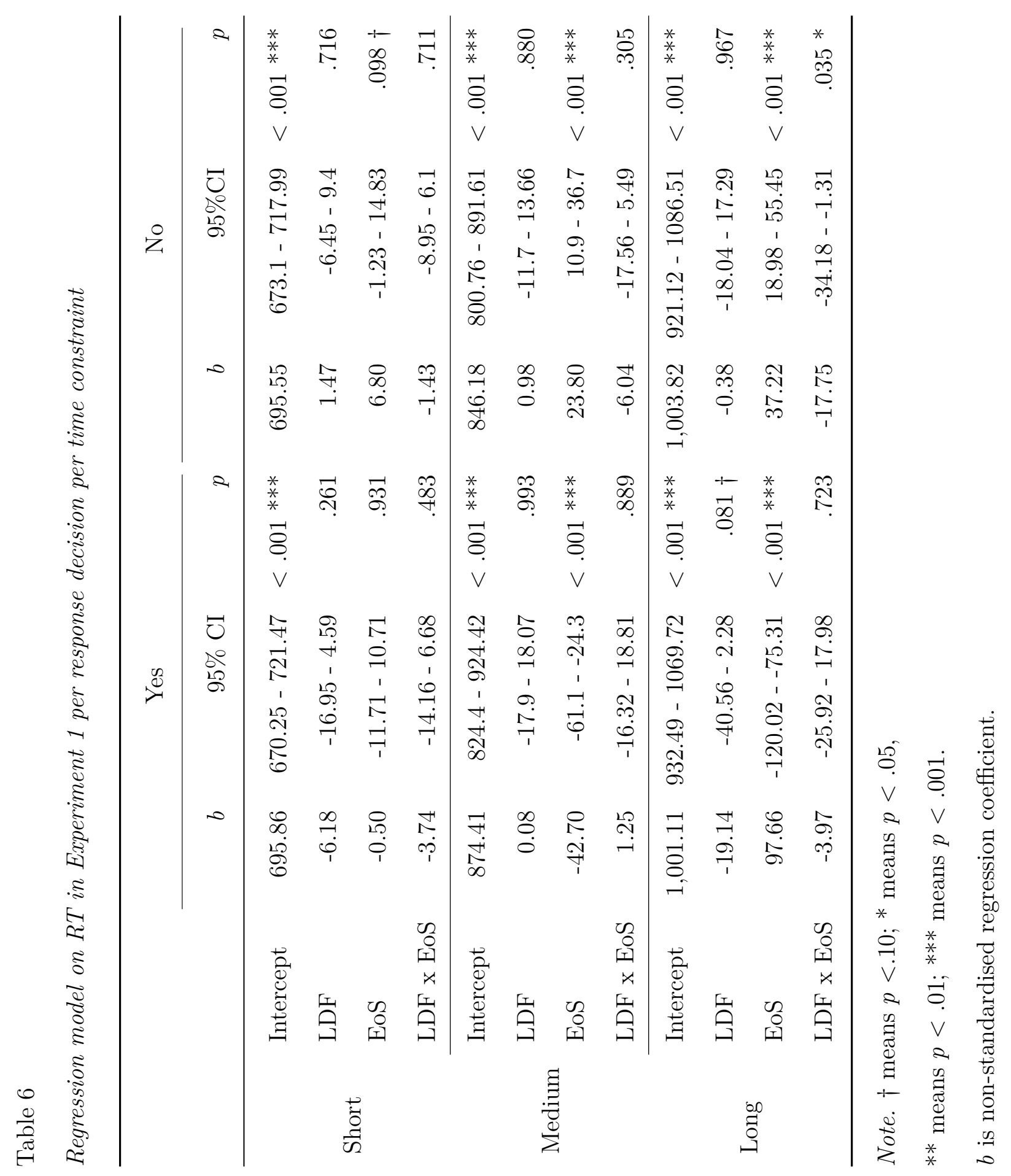




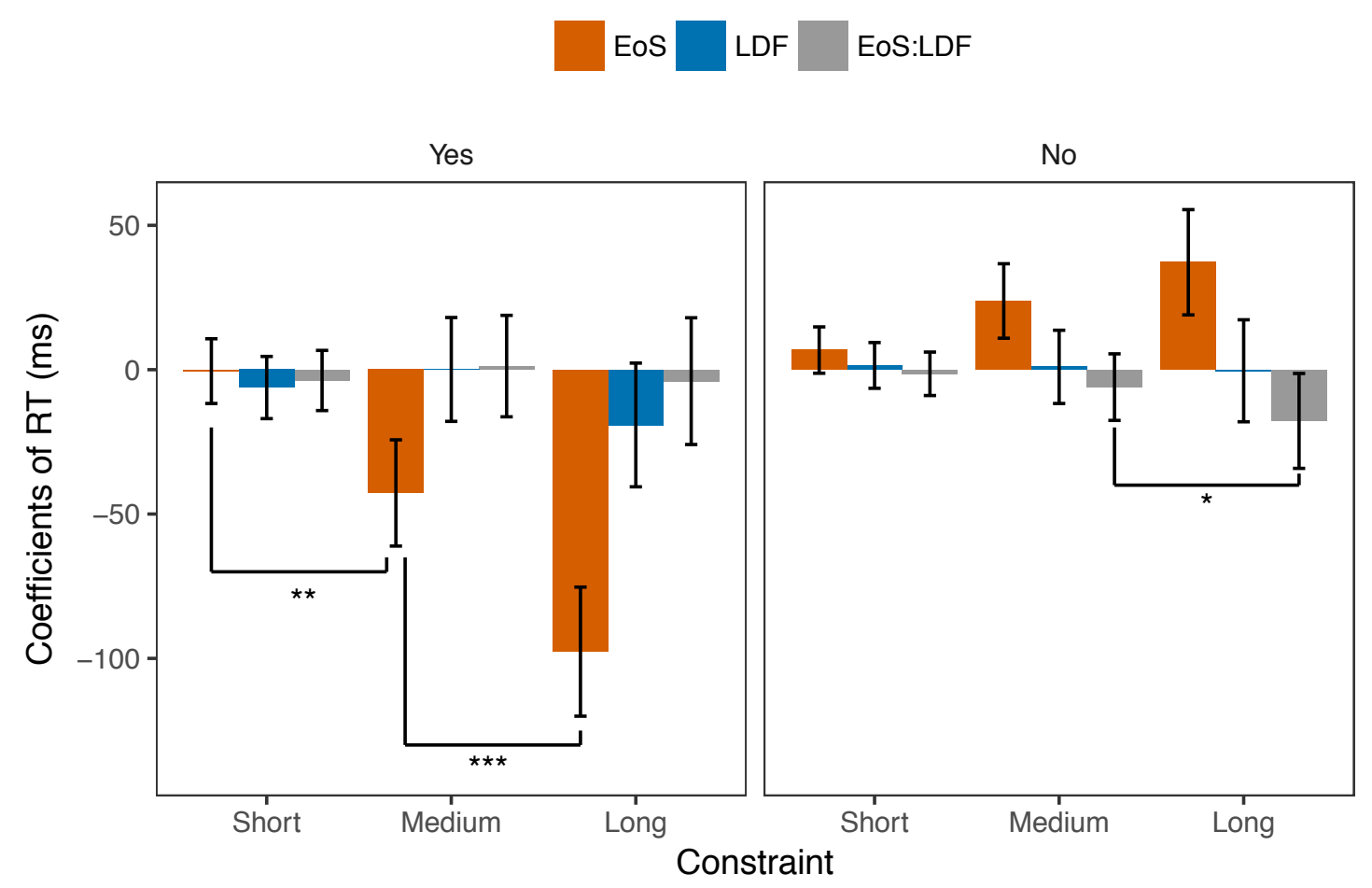

Figure 2. Regression coefficients of RT per response decision in Experiment 1. Error bars stand for $95 \%$ CI. Asterisks stand for $p$-values of interaction between variables and time constraint. *: $p<.05, * *: p<.01: * * *: p<.001$.

For "no" response, EoS had a positive effect (when LDF was at its mean) which meant that the harder it was typically considered to simulate a metaphor, the faster people rejected the metaphor as nonsensical (Table 5). This effect did not change significantly with time constraint, which meant that although the effect of EoS seemed to have increased from short to long constraint (Table 6 and Figure 2), the difference was not detected by the way the contrast was coded. Besides, EoS was moderated by LDF, which further interacted with the the time constraints. Separate analyses per time constraint showed that the interaction between EoS and LDF was borne out by the long constraint. Figure 3 shows the changes of the effect of EoS at four quartiles of LDF (within each quartile, the number of trials were the same). It could be seen that when the LDF was at the lower end, EoS had a positive effect. In 
other words, when the constituent words rarely appeared in the same context, people were still faster to reject a metaphor that was typically considered to be hard to simulate, and slower to reject one that was typically considered easy to simulate. As LDF increased, the effect of of EoS diminished and then turned to the opposite direction, so much so that when the constituent words frequently co-occurred, people were faster to reject the metaphors which were typically considered easy to simulate (though only a small number of trials were rejected in this case), but slower to reject those metaphors which were typically considered hard to simulate.
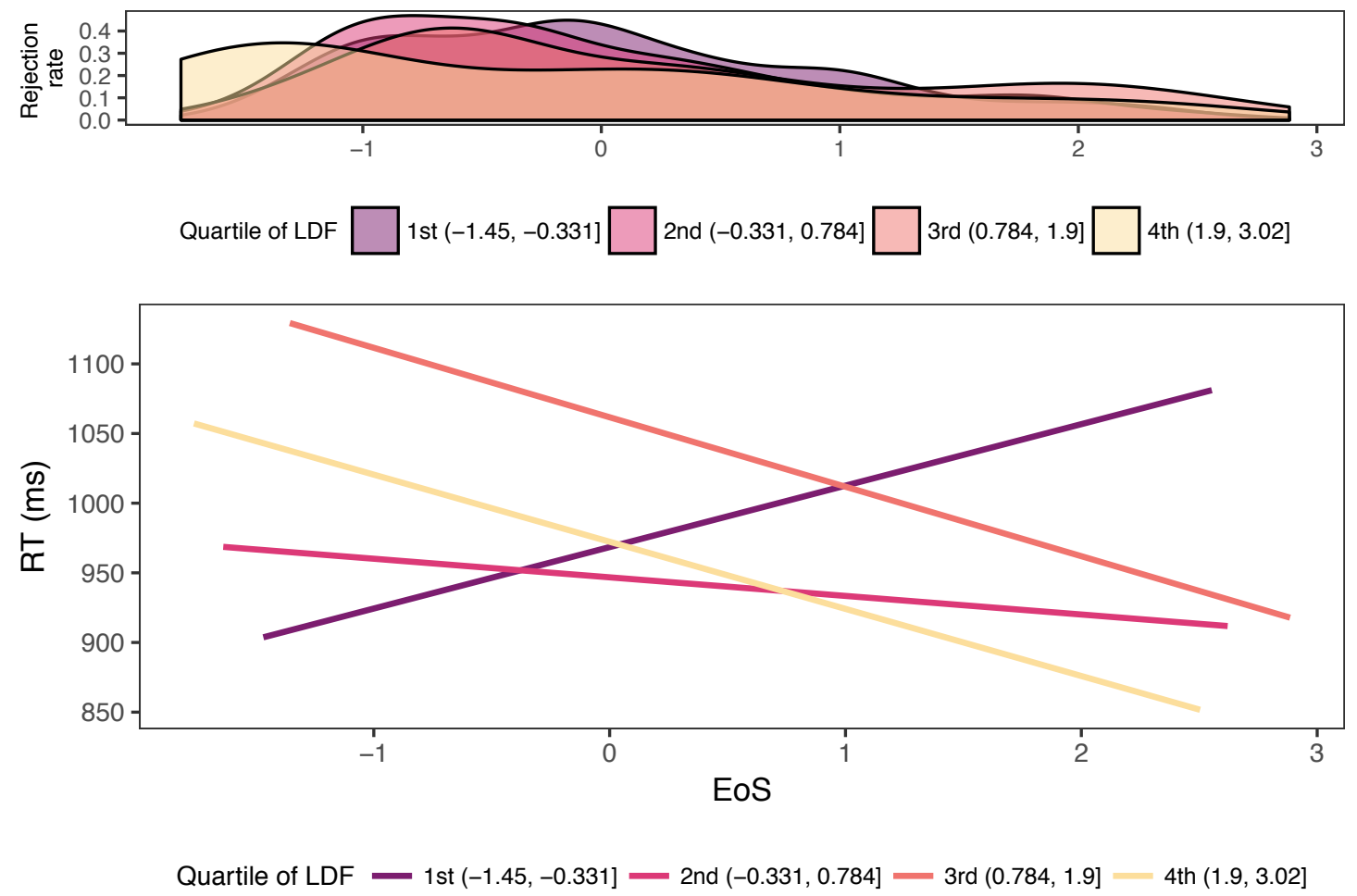

Figure 3. Effects of EoS at long constraint at four quartiles of LDF. As LDF increased from the 1st quartile (yellow) to the 4th quartile (violet), the effect of EoS changed from positive to negative. The density plot on the top shows the rejection rate along EoS per quartile of LDF. The rejection rate at the high end of EoS was less than $10 \%$. 
Summary. In this experiment, we found that EoS affected metaphor processing as predicted. When a metaphor was typically regarded to be easy to simulate, it was easier (more likely and faster) for people to accept the metaphor as sensible, and harder (less likely and slower) to reject it as nonsensical. The effect of EoS on acceptance speed was moderated by the time constraint, that is: people relied on simulation more when there was longer time available. Thus, it supported the simulation-linguistic based conceptual representation which claimed that the embodied component was more costly and time-consuming, and required longer time to be fully engaged.

The effect of EoS on rejection speed was more complex. It had a positive effect as expected when LDF was at its mean or particularly low. In other words, people found it easier to reject a metaphor which were typically regarded as difficult to simulate. However, the effect of EoS diminished and even was reversed if the constituent words frequently co-occurred, in which case people spent longer time to process a metaphor that was typically harder to simulate (Supply can be bright) than one that was typically easy to simulate (Students can be bright - rarely rejected). In other words, people were willing to expend more effort on a metaphor before rejecting it, if the constituent words frequently co-occurred. Such an interaction between EoS and LDF supported the linguistic shortcut hypothesis. The distributional patterns acted as a shortcut for identifying metaphors that could potentially be processed successfully. Metaphors, which were considered hard to simulate, were rejected quickly if their constituent words rarely co-occurred, but slowly if the constituent words often co-occurred.

However, we did not find strong support for the linguistic shortcut hypothesis in terms of how the linguistic component behaved under time constraint. LDF, contrary to our predictions, actually had a larger effect at the long constraint. It was possible that imposing time constraints did not incentivise the linguistic 
shortcut, but rather caused people to change their processing strategies altogether. As a result, the RT of the short constraint could not be predicted by either of the key predictors, which suggested that people may have based their judgements on variables at the lexical level such as word length and lexical frequencies (even though these variables did not have a strong correlation with the RT overall).

\section{Experiment 2: Interpretation Generation Task}

In this study, people performed an interpretation generation task under three different time constraints, a short time constraint of 2 seconds, a medium time constraint of 5 seconds, and a long time constraint of 8 seconds. If they decided they could interpret the sentences, they would be required to type down the meaning of the sentence. We predicted that EoS should have an effect on the response decision and RT (higher EoS, more and faster acceptance; lower EoS, more and faster rejection), as well as LDF (higher LDF, more and faster acceptance; lower LDF, more and faster rejection). Furthermore, EoS would have larger effect under longer time constraints, while LDF would have smaller effect under longer time constraints.

\section{Participants}

Fifty-four participants were recruited for this experiment (age: 20.43 (4.38) years; male: 15; left-handed: seven). They participated in the study for 30 minutes for $£ 3.50$ or the equivalent of course credits. The sample size was pre-determined based on a minimum acceptance rate of $45 \%$, which would provide at least the same number of data points as Liu et al. (2017); because this acceptance rate was achieved, it was not necessary to test any extra participants. 


\section{Materials}

The same materials were used in this experiment as in Experiment 1. However, because participants were now required to type interpretations, we reduced the length of the item lists from 113 items to 75-76 items in order to reduce chances of fatigue. Thus, six item lists were generated in the same way as the pilot study and Experiment 1. Each participants saw each adjective only once and the distribution of EoS and LDF were equal among the six lists $\left(\right.$ EoS: $F_{(5,446)}=0.21, p$ $\left.=.96 ; \mathrm{LDF}: F_{(5,446)}=0.49, p=.78\right)$.

\section{Procedure}

The procedure was the same as Experiment 1 with the following exceptions. The short, medium and long time constraints lasted 2 seconds, 5 seconds, and 8 seconds, respectively. Participants were asked to respond "yes" if they could think of a meaning for the sentence, and "no" if they could not think of a meaning for the sentence. If participants responded "yes", they were required to type the meaning of the sentence at the next screen, with no time limit for typing.

\section{Design and Analysis}

Same as Experiment 1 for response decisions ${ }^{5}$ and RT. ${ }^{6}$

\footnotetext{
5 The inclusion of participants in logistic mixed-effect model model as a random factor improved model fit above the empty model, $\chi^{2}(1)=562.71, \mathrm{p}<.001$, as did the inclusion of items as a crossed random factor above the participants-only model, $\chi^{2}(1)=738.94, p<.001$.

6 The inclusion of participants in linear mixed-effect model as a random factor improved model fit above the empty model, $\chi^{2}(1)=764.04, p<.001$. The inclusion of items as a crossed random factor did not improve model fit above the participants-only model $\chi^{2}(1)=0.00, p=1$. However, in order to keep the models consistent, both participants and items were included as crossed random factors in the linear models of RT as well as in the logistic models of response decision.
} 


\section{Results and Discussion}

The data exclusions were the same as in Experiment 1 with an additional validity check on participants' interpretations to make sure they were performing the task properly. An interpretation was marked as invalid if it was left blank, marked out as a mistake ("I don't know"), if it was clearly misread (interpretation specifies meaning of similar word, e.g. "charge" for "change") or simply replaced the adjectives with a synonym (not pre-registered but same with Liu et al. (2017), "Students can be bright" as "Students can be shiny"). All participants had a mean RT within three standard deviations of the overall mean, and had at least $50 \%$ of their interpretation marked as valid, and so all were included in the analysis. Ninety-nine trials were missed (no response), five trials were excluded as motor errors, 94 trials were marked as invalid meanings. In addition, 20 trials were outliers whose RT was 3SD from participants means per response decision and time constraint: that is for "yes" response, none for short constraint, $0.57 \%$ for medium constraint, and $0.36 \%$ for long constraint; for "no" response, $0.65 \%$ for short constraint, $0.76 \%$ for medium constraint, and $0.53 \%$ for long constraint. Consequently, 3853 trials were used in analysis: 1553 valid trials for "yes" response, and 2300 for "no" response (acceptance rate 39.30\%). For short constraint, the acceptance rate was $36.37 \%$; for mid constraint, $39.46 \%$; and for long constraint, $41.77 \%$.

Response decision. Logistic mixed-effect regression showed only an effect of EoS both in the overall analysis (Table 7) and analyses separated by time constraints (Table 8). As EoS increased, the decision to accept a metaphor as interpretable increased. The odds ratios were 3.97 for the overall analysis, 4.04 under the short constraint, 4.11 under the medium constraint, and 4.02 under the long constraint. Time constraints did not interact with either EoS or LDF. 
Table 7

Logistic mixed-effect regression of response decision in Experiment 2

\begin{tabular}{lccc}
\hline & $b$ & $95 \%$ CI & $p$ \\
\hline Intercept & -0.56 & $-0.97--0.14$ & $.009 * *$ \\
Long - medium & 0.14 & $-0.07-0.35$ & .203 \\
Medium - short & 0.08 & $-0.14-0.3$ & .464 \\
LDF & 0.04 & $-0.07-0.16$ & .484 \\
EoS & 1.38 & $1.25-1.51$ & $<.001 * * *$ \\
Long - medium x LDF & 0.02 & $-0.21-0.25$ & .889 \\
Medium - short x LDF & -0.04 & $-0.27-0.2$ & .758 \\
Long - medium x EoS & 0.01 & $-0.23-0.25$ & .916 \\
Medium - short x EoS & -0.05 & $-0.29-0.2$ & .704 \\
LDF x EoS & -0.01 & $-0.13-0.1$ & .821 \\
Long - medium x LDF x EoS & 0.09 & $-0.14-0.32$ & .44 \\
Medium - short x LDF x EoS & -0.01 & $-0.24-0.22$ & .919 \\
\hline
\end{tabular}

Note. $\dagger$ means $p<.10 ; *$ means $p<.05$;

** means $p<.01 ; * * *$ means $p<.001$.

$b$ is non-standardised regression coefficient. 
Table 8

Logistic regression model of response decision per time constraint in Experiment 2

\begin{tabular}{llrrr}
\hline & & $b$ & $95 \%$ CI & $p$ \\
\hline \multirow{4}{*}{ Short } & Intercept & 0.65 & $-1.1--0.21$ & $.004 * *$ \\
& LDF & 0.06 & $-0.11-0.24$ & .455 \\
& EoS & 1.40 & $1.17-1.62$ & $<.001 * * *$ \\
& LDF x EoS & -0.06 & $-0.23-0.1$ & .458 \\
\cline { 2 - 5 } Medium & Intercept & -0.60 & $-1.03--0.18$ & $.005 * *$ \\
& LDF & 0.02 & $-0.17-0.2$ & .860 \\
& EoS & 1.42 & $1.18-1.65$ & $<.001 * * *$ \\
& LDF x EoS & -0.05 & $-0.23-0.13$ & .568 \\
\cline { 2 - 5 } Long & -0.43 & $-0.89-0.04$ & $.072 \dagger$ & \\
& LDF & 0.10 & $-0.07-0.27$ & .267 \\
& EoS & 1.39 & $1.17-1.61$ & $<.001 * * *$ \\
& LDF x EoS & 0.01 & $-0.16-0.18$ & .908 \\
\hline
\end{tabular}

Note. $\dagger$ means $p<.10 ;{ }^{*}$ means $p<.05$;

** means $p<.01 ; * * *$ means $p<.001$.

$b$ is non-standardised regression coefficient. 
Response times. The overall mean RT were $1334 \mathrm{~ms}(S D=790 \mathrm{~ms})$ for "yes" responses, and $1120 \mathrm{~ms}(S D=653 \mathrm{~ms})$ for "no" responses. Separated by time constraint, the mean RT for "yes" responses was 1026ms ( $S D=322 \mathrm{~ms})$ for short constraint, $1239 \mathrm{~ms}(S D=631 \mathrm{~ms})$ for medium constraint, and 1698ms $(S D=$ $1037 \mathrm{~ms}$ ) for long constraint; the mean RT for "no" response was 965ms ( $S D=$ $344 \mathrm{~ms})$ for short constraint, $1082 \mathrm{~ms}(S D=567 \mathrm{~ms})$ for medium constraint, and $1318 \mathrm{~ms}(S D=886 \mathrm{~ms})$ for long constraint.

Omnibus linear mixed-effect regression showed (Table 9) EoS had a significant positive effect for "no" response on the RT regardless of LDF or time constraints, which was reversed for "yes" response. The effect was larger for "yes" than "no" response. The effect of LDF was contained in its interaction with response with a negative term, showing that although the effect of LDF was not significant for "no" response, it was indeed opposite for "yes" and "no" responses and was larger for "yes" response than "no" response.

After the omnibus analysis, the RT data was split first by response decisions and then by time constraint, same as Experiment 1. When separated by response decisions (Table 10), EoS had a negative effect on "yes" RT. That is, the easier it was considered to simulate a metaphor, the faster people accepted it as interpretable. Also, the effect of LDF was negative too at medium and long constraints (i.e., the higher the LDF was, the faster people accepted the metaphor as interpretable). Both effects were as predicted. EoS's effect did not increase significantly as the time constraints became longer, but only showed a slight trend between short and medium constraints (Table 11 and Figure 4). LDF interacted with time constraints between medium and long constraints, that is: the effect of LDF was larger at the long constraint than the medium and the short constraints, which was the opposite of what was predicted by the linguistic shortcut hypothesis. 
Table 9

Omnibus linear mixed-effect model on RT in Experiment 2

\begin{tabular}{|c|c|c|c|}
\hline & $b$ & $95 \% \mathrm{CI}$ & $p$ \\
\hline Intercept & $1,174.99$ & $1083.89-1266.08$ & $<.001 * * *$ \\
\hline Long - medium & 278.95 & $214.25-343.65$ & $<.001 * * *$ \\
\hline Medium - short & 94.56 & $30.66-158.46$ & $.004 * *$ \\
\hline Response & 179.22 & $130.63-227.82$ & $<.001 * * *$ \\
\hline $\mathrm{LDF}$ & 12.79 & $-15.5-41.08$ & .376 \\
\hline EoS & 45.63 & $15.54-75.72$ & $.003^{* *}$ \\
\hline Long - medium x Response & 153.87 & $49.07-258.66$ & $.004^{* *}$ \\
\hline Medium - short x Response & 158.39 & $51.06-265.71$ & $.004^{* *}$ \\
\hline Long - medium x LDF & -26.79 & $-92.81-39.24$ & .427 \\
\hline Medium - short x LDF & 29.89 & $-36-95.79$ & .374 \\
\hline Response $\mathrm{x}$ LDF & -51.63 & $-95.35--7.91$ & $.021 *$ \\
\hline Long - medium x EoS & 41.46 & $-28.48-111.41$ & .245 \\
\hline Medium - short x EoS & -25.86 & $-94.27-42.55$ & .459 \\
\hline Response x EoS & -194.84 & $-239.83--149.85$ & $<.001 * * *$ \\
\hline LDF x EoS & -18.41 & $-45.81-9$ & .188 \\
\hline Long - medium x Response x LDF & -13.21 & $-120.08-93.67$ & .809 \\
\hline Medium - short x Response x LDF & 8.69 & $-98.76-116.13$ & .874 \\
\hline Long - medium x Response $\mathrm{x}$ EoS & -86.62 & $-192.41-19.17$ & .109 \\
\hline Medium - short x Response x EoS & -50.12 & $-157.34-57.1$ & .360 \\
\hline Long - medium x LDF x EoS & -0.78 & $-64.34-62.78$ & .981 \\
\hline Medium - short x LDF x EoS & -23.22 & $-85.74-39.31$ & .467 \\
\hline Response x LDF x EoS & 37.61 & $-4.03-79.25$ & $.077 \dagger$ \\
\hline Long - medium x Response x LDF x EoS & -56.53 & $-155.62-42.56$ & .264 \\
\hline Medium - short x Response x LDF x EoS & 25.92 & $-73.3-125.14$ & .609 \\
\hline
\end{tabular}

Note. $\dagger$ means $p<.10 ;{ }^{*}$ means $p<.05$; 


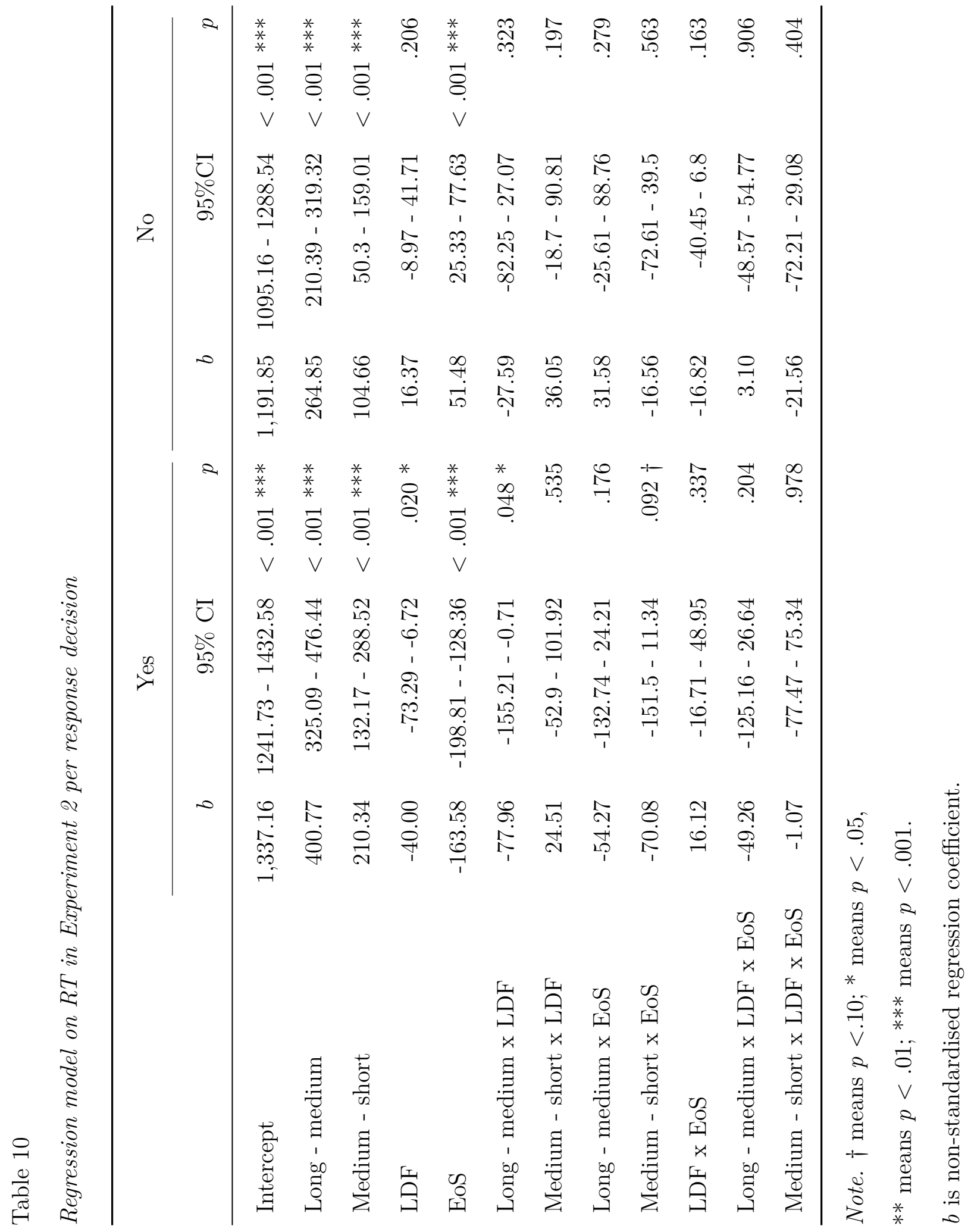


For "no" RT, only EoS had a strong and consistent effect (Table 10). The harder it was considered to simulate a metaphor, the faster people rejected it as uninterpretable. The effect held true for all levels of time constraints. In contrast, LDF did not have an effect at all on "no" RT (Table 11 and Figure 4). 


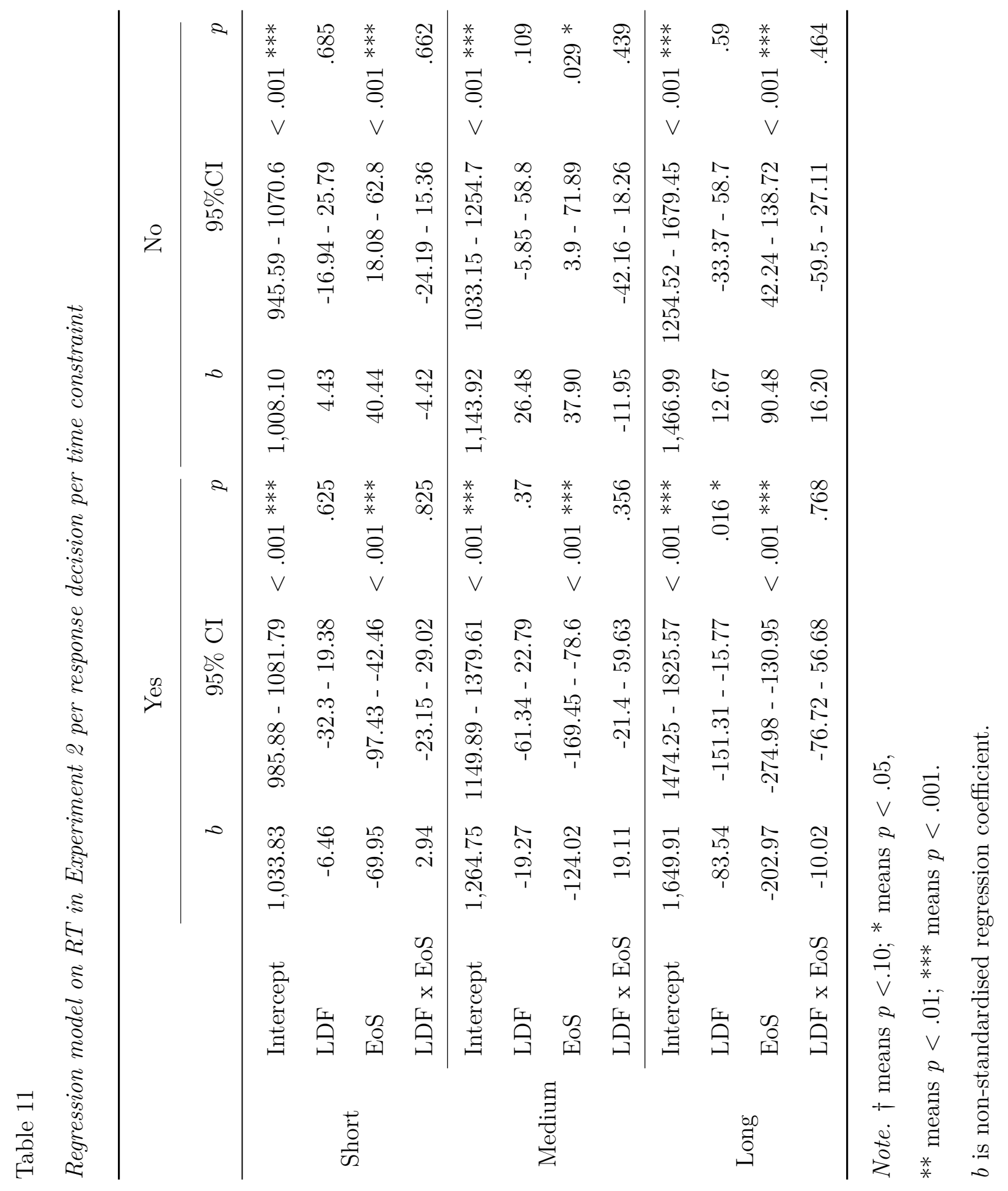




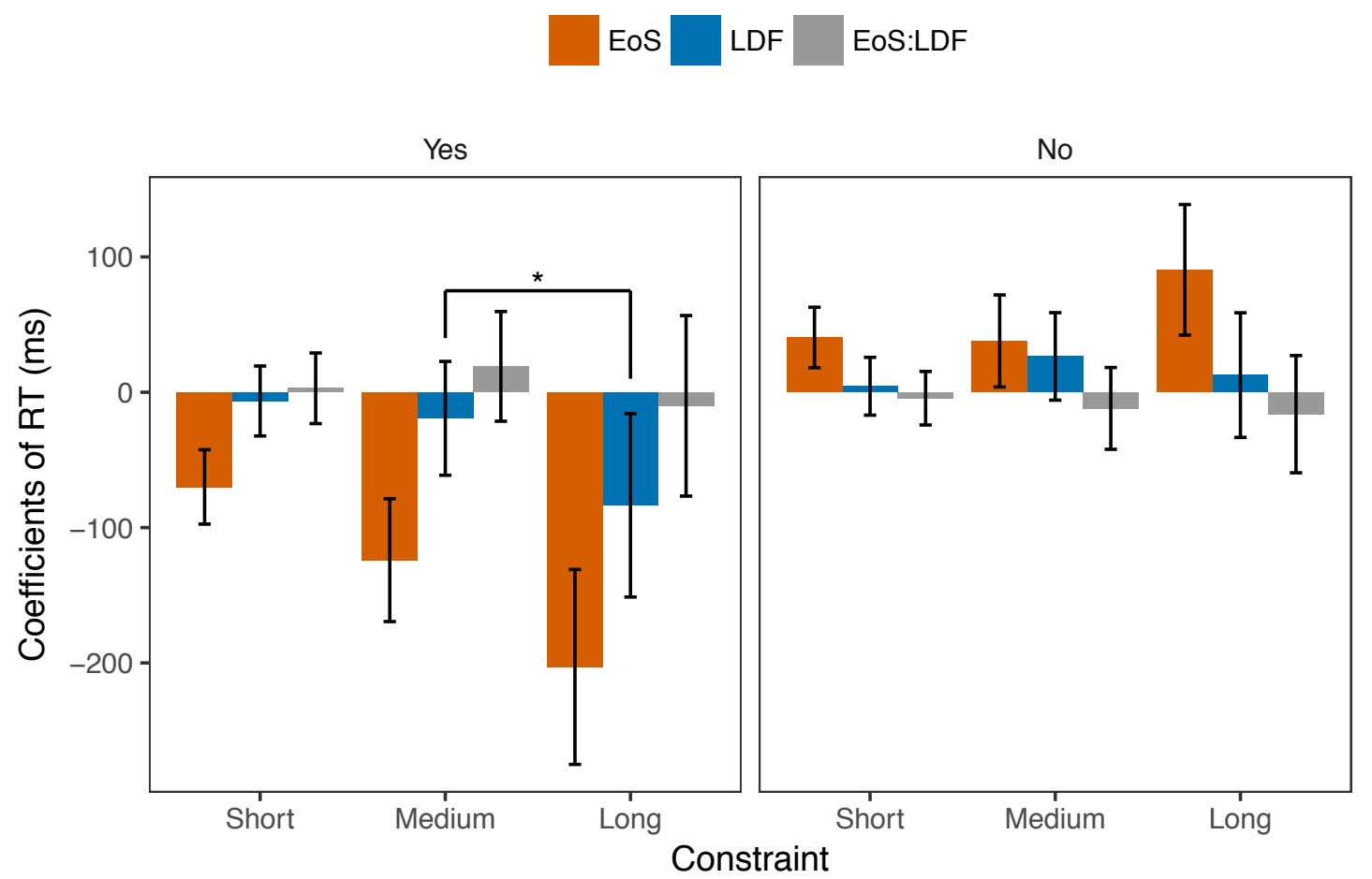

Figure 4. Regression coefficients of RT per response decision in Experiment 2 (i.e. interpretation generation). Error bars stand for 95\% CI. Asterisks stand for $p$-values of interaction between variables and time constraint. ${ }^{*}: p<.05$.

Summary. EoS had a significant effect on response decision and both "yes" and "no" RT as predicted and demonstrating a similar pattern to the sensibility judgement task in Experiment 1. The easier it was to simulate a metaphor, the more likely people accepted it as interpretable, the faster they could think of a meaning, and the longer it took to reject as uninterpretable. Although EoS trended toward the prediction of having a greater effect at longer time constraint, the effect was not reliable. The lack of the time-constraint effect may simply be a result of the coding scheme adopted by my present study. As we always contrasted the two closest time constraints in both experiments (i.e., difference between long and medium constraints, difference between medium and short constraints), the coding could be overly conservative. If the long constraint could be contrasted with the 
short constraint, we might have found a significant increase of the effect of EoS in this experiment. Further examination of the data could address this problem (which could not be done for the lack of time).

On the other hand, the effects of LDF were mixed. As predicted, LDF had a negative effect on "yes" RT: that is, when words in a metaphor often co-occurred, people were faster to accept it as interpretable. However, there was no evidence that shorter time constraints boosted the effect of LDF, contrary to our prediction, and the effect of LDF was instead reduced at shorter time constraints. Finally, LDF had no effect on response decisions nor on "no" RTs.

\section{Cross-Experiment Comparison}

To test our cross-experiment hypotheses, we conducted a meta-analysis of the data from Experiments 1 and 2. We expected EoS to have a larger effect for deep interpretation generation than for shallow sensibility judgement, while the effect of LDF would be the other way round.

\section{Method}

The data from Experiments 1 and 2 were combined for analysis: response decision in logistic mixed-effects regression, and response time in linear mixed-effects regression. Task was employed as a categorical variable (coded 0 for sensibility judgement and 1 for interpretation generation). In analysis of response decision, EoS and LDF from two datasets were first combined and then centred, while in analysis of response time, they were first combined and then orthogonalised. Levels of time constraint were also included in the models with backward contrast coding, similar to Experiments 1 and 2: that is, Contrast 1 referred to long constraint minus medium constraint (regardless of tasks), and Contrast 2 referred to medium minus short constraint (regardless of tasks). 


\section{Results and Discussion}

Response decision. Results of the logistic mix-effect model was shown in Table 12. Task enhanced the effect of EoS when LDF was at its mean, such that EoS had a larger, more positive effect in the deep task than the shallow task. That is, people were more likely to accept a metaphor that was typically considered easy to simulate, and even more likely to accept such a metaphor in the deep interpretation generation task than in the shallow sensibility judgement task. EoS was also moderated by time constraint during the shallow task, such that it had a larger, more positive effect at medium time constraint than short time constraint (as predicted); but this moderation effect of time constraint did not appear during the deep task, such that, in the deep task, the effect of EoS did not increase because of time constraint. Finally, LDF had little effect on response decision with a non-significant trend for LDF positively affect chance of acceptance at higher levels of EoS.

Response time. Since the predictors had effects in opposite directions for "yes" and "no" responses, we analysed RT separated by the response (Table 13). For both "yes" and "no" responses, EoS had a larger effect for the deep task than the shallow task. In other words, people relied on embodied simulation more during deeper processing than shallower processing as predicted, which held true across the time constraints. The effect of LDF appeared for "yes" response regardless of EoS, but this effect was conditional to task. That is, LDF only had a significant effect in the deep interpretation generation task but not in the shallow sensibility judgement task, which was opposite to the hypothesis that shallow processing should encourage the using of the linguistic shortcut. Apart from this effect, LDF did not have any other effects. It did not affect "no" RT. Nor was it affected by time constraints. All these null effects did not support the hypotheses concerning the linguistic component. 
Table 12

Logistic mixed-effect regression of response decision for cross-experiment comparison

\begin{tabular}{|c|c|c|c|}
\hline & $b$ & $95 \% \mathrm{CI}$ & $p$ \\
\hline Intercept & -0.65 & $-1--0.3$ & $<.001 * * *$ \\
\hline Task & 0.12 & $-0.37-0.6$ & .64 \\
\hline Long - medium & -0.01 & $-0.18-0.16$ & .908 \\
\hline Medium - short & -0.01 & $-0.18-0.16$ & .922 \\
\hline $\mathrm{LDF}$ & 0.06 & $-0.03-0.15$ & .177 \\
\hline EoS & 1.18 & $1.09-1.28$ & $<.001 * * *$ \\
\hline Task x long - medium & 0.14 & $-0.13-0.41$ & .310 \\
\hline Task x medium - short & 0.09 & $-0.18-0.36$ & .519 \\
\hline Task x LDF & -0.01 & $-0.13-0.1$ & .817 \\
\hline Long - medium $\mathrm{x}$ LDF & -0.08 & $-0.26-0.1$ & .389 \\
\hline Medium - short x LDF & -0.01 & $-0.19-0.18$ & .926 \\
\hline Task x EoS & 0.13 & $0-0.26$ & $.043 *$ \\
\hline Long - medium x EoS & -0.12 & $-0.31-0.07$ & .231 \\
\hline Medium - short x EoS & 0.38 & $0.19-0.57$ & $<.001 * * *$ \\
\hline LDF x EoS & 0.08 & $-0.01-0.17$ & $.081 \dagger$ \\
\hline Task x long - medium x LDF & 0.08 & $-0.21-0.37$ & .567 \\
\hline Task x medium - short x LDF & -0.02 & $-0.31-0.27$ & .885 \\
\hline Task x long - medium x EoS & 0.12 & $-0.18-0.42$ & .418 \\
\hline Task x medium - short x EoS & -0.42 & $-0.73--0.12$ & $.006^{* *}$ \\
\hline Task x LDF x EoS & -0.09 & $-0.21-0.03$ & .143 \\
\hline Long - medium x LDF x EoS & -0.02 & $-0.21-0.16$ & .816 \\
\hline Medium - short x LDF x EoS & -0.04 & $-0.22-0.15$ & .687 \\
\hline Task x Long - medium x LDF x EoS & 0.12 & $-0.17-0.41$ & .419 \\
\hline Task x Medium - short x LDF x EoS & 0.02 & $-0.27-0.31$ & .877 \\
\hline
\end{tabular}

Note. $\dagger$ means $p<.10 ;{ }^{*}$ means $p<.05$; 


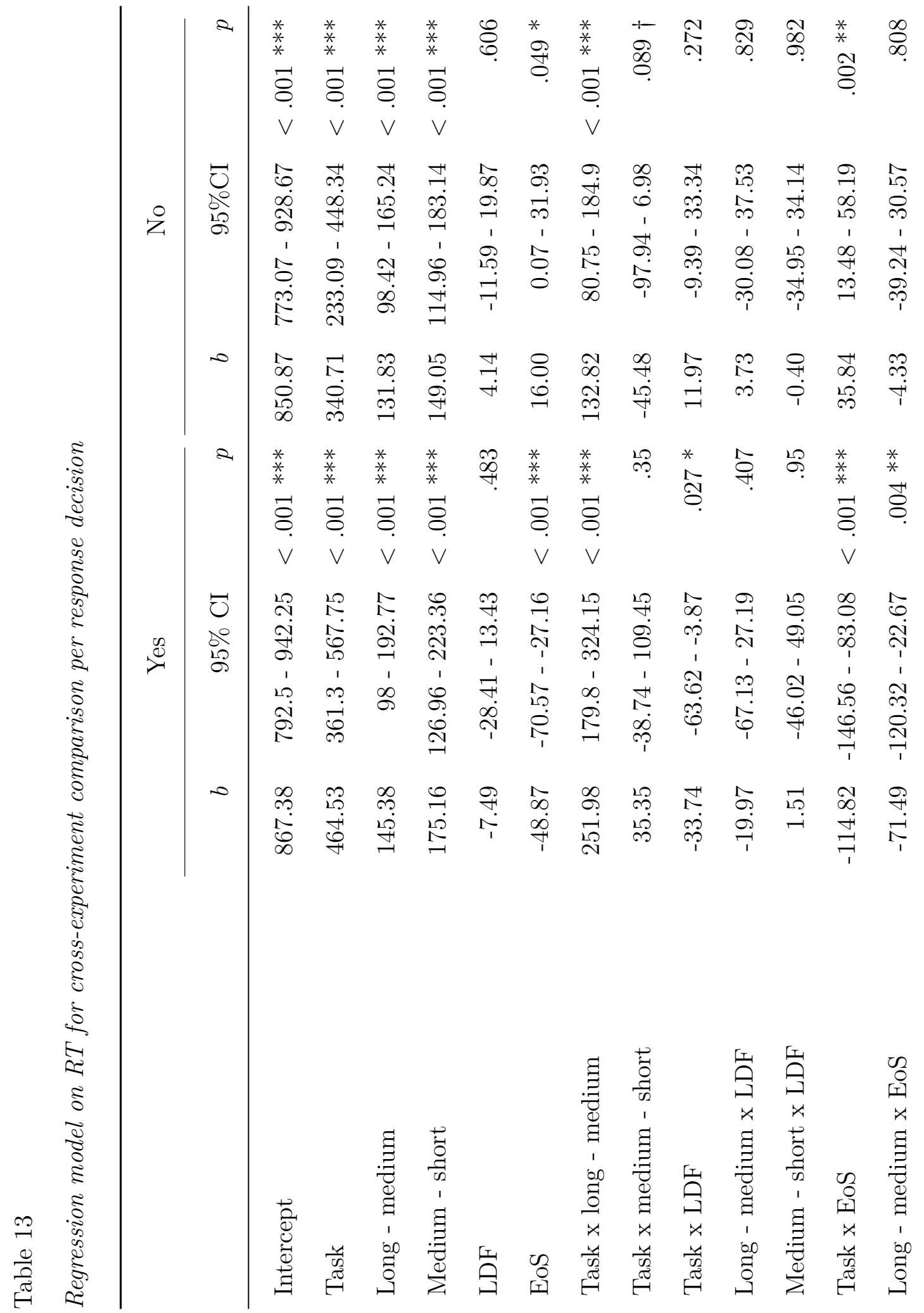




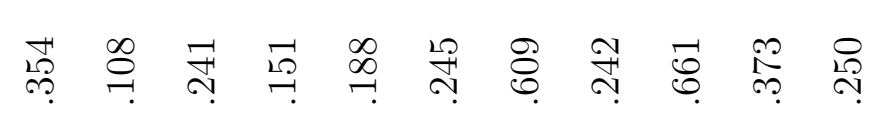

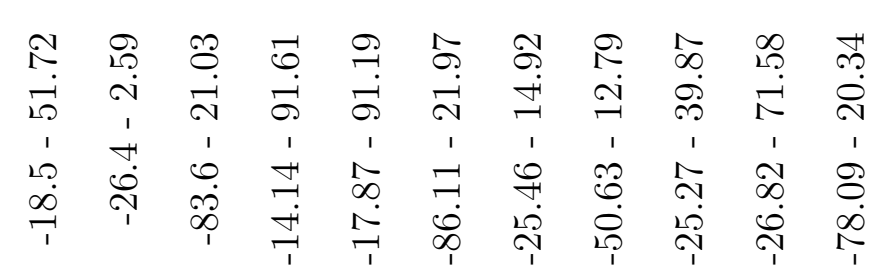

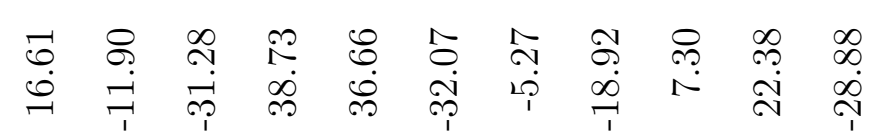

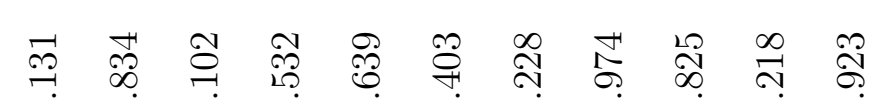

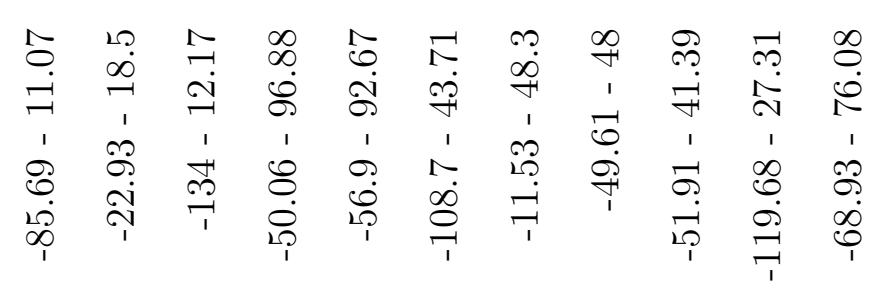

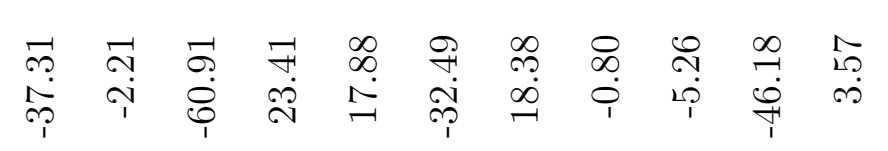

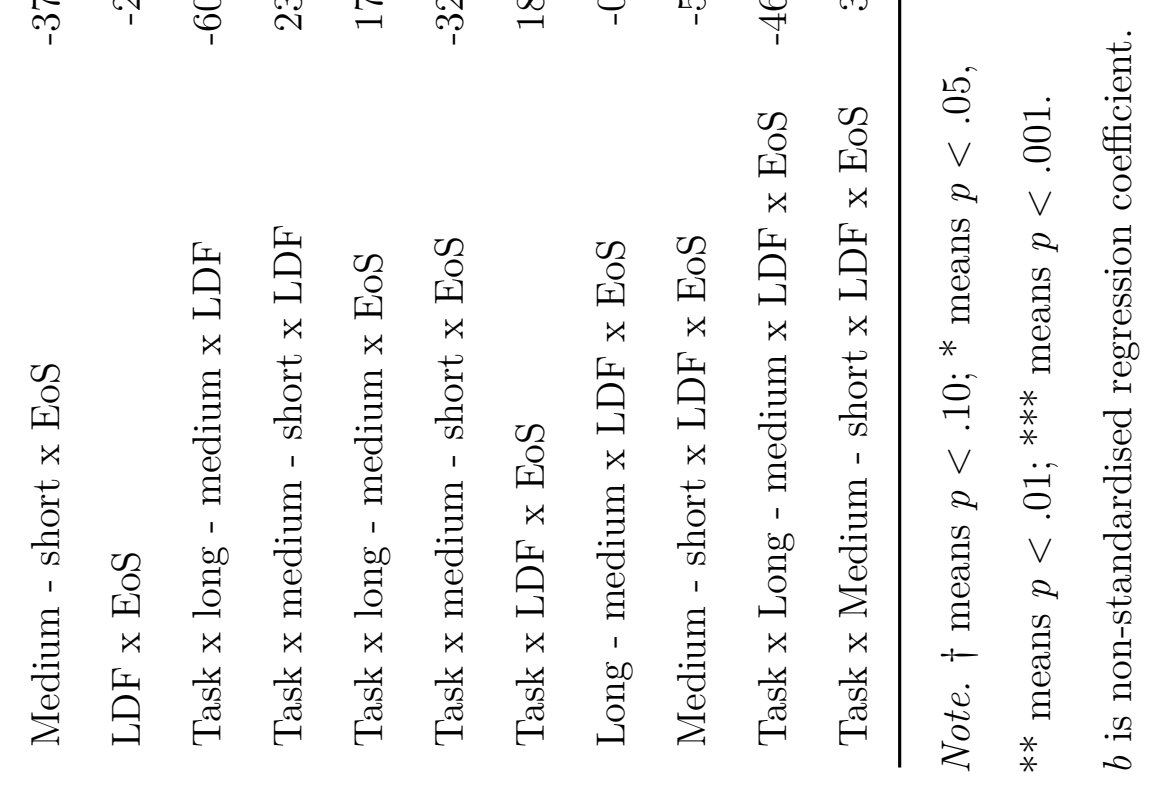




\section{General Discussion}

The current study was conducted to replicate the previous study on the conceptual representation during metaphor processing and answer the specific question, whether limited time resources would affect the conceptual representation. Our findings confirmed the hypothesis that both embodied and linguistic components existed as parts of a conceptual representation. The embodied component played a prominent and consistent role in the representation of meaning. The easier a metaphor was considered to simulate, the easier it was to accept it as either sensible or interpretable; the harder it was considered to simulate, the easier it was to reject it. We also found, as predicted, that the embodied component was more engaged during the deep processing of interpretation generation than during the relatively shallower processing of sensibility judgement, and it was also generally more engaged when there was more processing time available. Thus, it confirmed the suggestion of grounded representation that the embodied component was more costly and time-consuming (Barsalou et al., 2008; Connell \& Lynott, 2014).

In contrast, evidence for the linguistic component and the linguistic shortcut hypothesis was mixed. We found evidence that the linguistic component affected metaphor processing independently of ease of simulation. The more often two constituent words of a metaphor co-occurred, the easier (at least faster) it was to accept it as interpretable (which was not found for shallow sensibility judgement). However, the linguistic component did not have a greater effect when the time resources were restricted as we hypothesised. In fact, we found that people were more reliant on the linguistic component when they had a longer interval for response. 


\section{Complex Processing Strategy during Metaphor Processing}

The surprising results suggested that people have adopted a processing strategy more complex than we originally predicted. We introduced the time constraints to put people under pressure so as to incentivise them to use the linguistic shortcut, which would sacrifice the accuracy of processing in return for speed. It was clear that people were put under pressure because they adopted a very conservative processing strategy. Their acceptance rate was lower than the previous study, and even when the time constraints were relaxed, their acceptance rate did not increase noticeably. However, people were unwilling to take the speed-accuracy tradeoff. This could be a unique phenomenon to metaphor processing. Firstly, distributional patterns of constituent words are not often reliable during metaphor processing, because it is common to read metaphors that are perfectly sensible and meaningful whose constituent words rarely co-occur (e.g., abrasive personality, lukewarm supporters). Therefore, people might deem the sacrifice of accuracy to be too great if they were to take the linguistic shortcut. Secondly, metaphors may encourage people to engage in the simulation of affective experiences more than their literal counterparts (Citron \& Goldberg, 2014). As a result, people put a much heavier reliance on the embodied component than during literal language processing. Having the time constraints meant that full engagement of embodied simulation was not always possible. Therefore, people might have switched their processing strategies altogether to focus on the lower-level lexical variables such as lexical frequencies or word lengths.

When the time allowed, embodied and linguistic components affected metaphor processing mutually in a complex interplay with each other. During shallow processing, people primarily relied on embodied simulation to decide whether a metaphor was sensible or not. However, before the embodied component 
was fully engaged, the linguistic component was able to signal whether the processing was likely to succeed. This linguistic information was treated very carefully because, as discussed above, the linguistic distributional patterns were not reliable for metaphor processing. Therefore, although the linguistic shortcut hypothesis suggested that metaphor could be rejected quickly if the constituent words rarely co-occurred, people would still wait for more information to come in from the embodied component. Only when the distributional frequency was very high would people rely on it to guide their future processing. In other words, when distributional frequency was low, people were not willing to take the linguistic shortcut to conserve energy; but when distributional frequency was high, people were willing to expend more effort on embodied simulation. As a result of such a complex interplay, the counter-intuitive pattern of results was found: people could sometimes (very occasionally) reject a metaphor quickly even if the distributional frequency was high and simulation was typically considered easy (e.g., Students can be bright), but they would take a long time to reject a metaphor that was considered hard to simulate if distributional frequency was high (e.g., Supply can be bright). In other words, embodied and linguistic components posed a conflict, and people were reluctant to reject a metaphor when either components suggested that the processing could be successful.

During deep processing, the influence of the two components were more straightforward. People still primarily based their judgements on simulation, but people also could judge a metaphor as interpretable based on the linguistic distributional patterns alone. When the distributional frequency was high which suggested that the processing was going to be successful (e.g., Students can be bright or Supply can be bright), people accepted such a metaphor more quickly. 


\section{Effects of Tasks}

Contrary to the linguistic shortcut hypothesis, it was the deeper processing that encouraged the reliance on the linguistic component (same as Liu et al., 2017). It might be explained by the use of the linguistic shortcut to conserve resources. Because the deeper processing was more computationally heavy, the linguistic shortcut could conserve more resources during deep processing, so the deeper processing actually incentivised the use of the linguistic shortcut instead. However, although the linguistic component directly affected the response during deep processing, it did not take the place of simulation. In fact, regardless of the distributional pattern, the embodied component had a prominent effect, which did not interact with EoS and was not affected by the time constraints either. These findings all suggested that people used the embodied component to construct a coherent conceptual representation in deep processing even when they used the linguistic component as a shortcut.

The processing model based on the current study is different from previous proposals of the linguistic shortcut hypothesis in a significant way. The measures introduced to encourage the use of the linguistic shortcut (i.e., time constraints and shallow processing) do not enhance the effect of LDF in any way, but instead reduce and eliminate the effect. Therefore, although the linguistic component may have the potential to be a heuristic for conceptual representation, and is used as a shortcut to some extent, people do not fully rely on the it to produce good-enough representations or to conserve resources in all circumstances.

\section{Contributors to the Null Effects of the Linguistic Component}

In the present study, we did not find strong evidence for the linguistic shortcut hypothesis. One of the reasons may be that the linguistic distributional frequency 
was minimalist rendition of the linguistic component. LDF uses the n-gram distributional frequency, which measured how often two words co-occurred in the same context (e.g., solution and bright). It was different from other vector-based distributional models (e.g., latent semantic analysis) which measured how often words occurred in similar context. For example, although solution and bright do not co-occur frequently in the same context, thus having a low n-gram frequency, solution frequently co-occurred in similar contexts as words like question, answer and students. Therefore, the vector-based distributional models might better at capturing the distributional pattern for metaphor processing, which should be explored further in future studies.

There is one further gap to address before we are in a position to conclude on the role of the linguistic component. According to theories of combined simulation-linguistic conceptual representations, both linguistic and embodied components are activated immediately in language processing (Barsalou, 1999; Connell \& Lynott, 2014; Louwerse, 2011), but here we could only measure the end product of metaphor processing with RT and response decisions. LDF could have been activated and influential to processing from the beginning of a trial, but by the time that a response was made (at least $200 \mathrm{~ms}$ after the adjective onset), the initial shortcut effect might have faded. To fully examine the role of the linguistic component during online processing, measurements with high temporal resolution such as EEG and MEG should be taken.

\section{Conclusions}

In conclusion, we found that metaphor processing relies on the unique contributions of embodied simulation and linguistic distributional patterns. These two components both inform conceptual representation and could cause conflict when there is no agreement between them. Combined with previous research on 
metaphor processing, literal language processing and conceptual combination, these findings indicate that conceptual representation involves a complex interaction between the two components, and flexibly constructed based on the demand of the task. 


\section{References}

Allport, D. A. (1985). Distributed memory, modular subsystems and dysphasia. In S.

Newman \& R. Epstein (Eds.), Current perspectives in dysphasia (pp. 207-244).

Edinburgh: Churchill Livingstone. Retrieved from

https://www.scribd.com/document/17676263/Allport-1985-Distributed-Memory-

Modular-Subsystems-and-Dysphasia

http://scholar.google.com/scholar?hl=en $\{\backslash \&\}$ btnG $=$ Search $\{\backslash \&\} \mathrm{q}=$ intitle:Distributed + memory + ,

Andrews, M., Vigliocco, G., \& Vinson, D. (2009). Integrating experiential and

distributional data to learn semantic representations. Psychological Review, 116(3), 463-498. doi:10.1037/a0016261

Aslin, R. N., Saffran, J. R., \& Newport, E. L. (1998). Computation of Conditional Probability Statistics By 8-Month-Old Infants. Psychological Science, 9(4), 321-324.

Auguie, B. (2017). GridExtra: Miscellaneous functions for "grid" graphics. Retrieved from https://CRAN.R-project.org/package=gridExtra

Aust, F. (2016). Citr: 'RStudio' add-in to insert markdown citations. Retrieved from https://CRAN.R-project.org/package=citr

Aust, F., \& Barth, M. (2017). papaja: Create APA manuscripts with R Markdown. Retrieved from https://github.com/crsh/papaja

Bache, S. M., \& Wickham, H. (2014). Magrittr: A forward-pipe operator for $r$. Retrieved from https://CRAN.R-project.org/package=magrittr

Barr, D. J., Levy, R., Scheepers, C., \& Tily, H. J. (2013). Random effects structure for confirmatory hypothesis testing: Keep it maximal. Journal of Memory and Language, 68(3). doi:10.1016/j.jml.2012.11.001

Barsalou, L. W. (1999). Perceptual symbol systems. The Behavioral and Brain Sciences, 22(4), 577-609; discussion 610-60. Retrieved from http://www.pubmedcentral.nih.gov/articlerender.fcgi?artid=1693222\{\\&\}tool=pmcentrez $\{\backslash \&\}$ ren

Barsalou, L. W., Santos, A., Simmons, W. K., \& Wilson, C. (2008). Language and 
simulation in conceptual processing. In A. M. Glenberg \& A. C. Graesser (Eds.), Symbols, embodiment, and meaning (pp. 245-283). Oxford, England: Oxford University Press. Retrieved from http://psychology.emory.edu/cognition/barsalou/papers/Barsalou\{\_\}et $\left\{\backslash \_\right\} a l\left\{\backslash \_\right\} \operatorname{chap}\left\{\backslash \_\right\} 200$

Bartoń, K. (2017). MuMIn: Multi-model inference. Retrieved from https://CRAN.R-project.org/package=MuMIn

Bates, D., \& Maechler, M. (2017). Matrix: Sparse and dense matrix classes and methods. Retrieved from https://CRAN.R-project.org/package=Matrix

Bates, D., Mächler, M., Bolker, B., \& Walker, S. (2015). Fitting linear mixed-effects models using lme4. Journal of Statistical Software, 67(1), 1-48. doi:10.18637/jss.v067.i01

Bonner, M. F., \& Grossman, M. (2012). Gray matter density of auditory association cortex relates to knowledge of sound concepts in primary progressive aphasia. Journal of Neuroscience, 32(23), 7986-7991. doi:10.1523/JNEUROSCI.6241-11.2012

Boulenger, V., Mechtouff, L., Thobois, S., Broussolle, E., Jeannerod, M., \& Nazir, T. A. (2008). Word processing in Parkinson's disease is impaired for action verbs but not for concrete nouns. Neuropsychologia, 46(2), 743-756. doi:10.1016/j.neuropsychologia.2007.10.007

Brants, T., \& Franz, A. (2006). Web 1T 5-gram Version 1 LDC2006T13. DVD. Philadelphia: Linguistic Data Consortium.

Citron, F. M. M., \& Goldberg, A. E. (2014). Metaphorical Sentences Are More Emotionally Engaging than Their Literal Counterparts. Journal of Cognitive Neuroscience, 26(11), 2585-2595. doi:10.1162/jocn_a_00654

Cohen, J., Cohen, P., West, S. G., \& Aiken, L. S. (2003). Applied Multiple Regression/Correlation Analysis for the Behavioral Sciences. Mahwah, NJ: Erlbaum. Retrieved from https://books.google.co.uk/books?id=fAnSOgbdFXIC Connell, L., \& Lynott, D. (2013). Flexible and fast: linguistic shortcut affects both shallow and deep conceptual processing. Psychonomic Bulletin \& Review, 20(3), 542-50. 
doi:10.3758/s13423-012-0368-x

Connell, L., \& Lynott, D. (2014). Principles of representation: Why you can't represent the same concept twice. Topics in Cognitive Science, 6(3), 390-406. doi:10.1111/tops.12097

Dantzig, S. van, Cowell, R. a, Zeelenberg, R., \& Pecher, D. (2011). A sharp image or a sharp knife: norms for the modality-exclusivity of 774 concept-property items. Behavior Research Methods, 43(1), 145-54. doi:10.3758/s13428-010-0038-8

Dowle, M., \& Srinivasan, A. (2017). Data.table: Extension of 'data.frame'. Retrieved from https://CRAN.R-project.org/package=data.table

Ferreira, F., Bailey, K., \& Ferraro, V. (2002). Good-enough representations in language comprehension. Current Directions in Psychological Science, 11(1), 11-15.

Retrieved from http://cdp.sagepub.com/content/11/1/11.short

Gelman, A., \& Su, Y.-S. (2016). Arm: Data analysis using regression and multilevel/hierarchical models. Retrieved from https://CRAN.R-project.org/package=arm

Glenberg, A. M., \& Gallese, V. (2012). Action-based language: a theory of language acquisition, comprehension, and production. Cortex, 48(7), 905-22. doi:10.1016/j.cortex.2011.04.010

Goldberg, R. F., Perfetti, C. A., \& Schneider, W. (2006). Perceptual Knowledge Retrieval Activates Sensory Brain Regions. Journal of Neuroscience, 26(18), 4917-4921. doi:10.1523/JNEUROSCI.5389-05.2006

Henry, L., \& Wickham, H. (2017). Purrr: Functional programming tools. Retrieved from https://CRAN.R-project.org/package=purrr

Johns, B. T., \& Jones, M. N. (2012). Perceptual Inference Through Global Lexical Similarity. Topics in Cognitive Science, 4(1), 103-120. doi:10.1111/j.1756-8765.2011.01176.x

Kuznetsova, A., Brockhoff, P. B., \& Christensen, R. H. B. (2017). lmerTest package: Tests 
in linear mixed effects models. Journal of Statistical Software, 82(13), 1-26. doi:10.18637/jss.v082.i13

Liu, P. Q., Connell, L., \& Lynott, D. (2017). Can illness be bright? Metaphor comprehension depends on linguistic and embodied factors. In G. Gunzelmann, A. Hows, T. Tenbrink, \& E. J. Davelaar (Eds.), 39th annual conference of cognitive science society (pp. 2604-2609). Austin, TX: Cognitive Science Society. Retrieved from https://mindmodeling.org/cogsci2017/papers/0494/paper0494.pdf

Liu, P. Q., Connell, L., \& Lynott, D. (2018). Ease-of-Simulation norms for 452 Adjective Metaphors. Manuscript Under Review.

Louwerse, M. M. (2011). Symbol interdependency in symbolic and embodied cognition. Topics in Cognitive Science, 3(2), 273-302. doi:10.1111/j.1756-8765.2010.01106.x

Louwerse, M. M., \& Connell, L. (2011). A taste of words: linguistic context and perceptual simulation predict the modality of words. Cognitive Science, 35(2), 381-98. doi:10.1111/j.1551-6709.2010.01157.x

Louwerse, M. M., \& Jeuniaux, P. (2008). Language comprehension is both embodied and symbolic. In A. Glenberg \& A. C. Graesser (Eds.), Embodiment and meaning: A debate (pp. 309-326). Oxford, England: Oxford University Press. Retrieved from http://www.madresearchlab.org/Selected $\left\{\backslash \_\right\}$Publications $\left\{\backslash \_\right\}$files/LouwerseJeuniauxBookChapt

Lynott, D., \& Connell, L. (2009). Modality exclusivity norms for 423 object properties. Behavior Research Methods, 41(2), 558-64. doi:10.3758/BRM.41.2.558

Lynott, D., \& Connell, L. (2010). Embodied conceptual combination. Frontiers in Psychology, 1 (November), 212. doi:10.3389/fpsyg.2010.00212

Monaghan, P., Chang, Y.-N., Welbourne, S., \& Brysbaert, M. (2017). Exploring the relations between word frequency, language exposure, and bilingualism in a computational model of reading. Journal of Memory and Language, 93, 1-21. doi:10.1016/J.JML.2016.08.003

Morey, R. D., \& Rouder, J. N. (2015). BayesFactor: Computation of bayes factors for 
common designs. Retrieved from

https://CRAN.R-project.org/package=BayesFactor

Niedenthal, P. M. (2007). Embodying Emotion. Science, 316(5827), 1002-1005.

doi:10.1126/science.1136930

Nowosad, J. (2017). Rcartocolor: 'CARTOColors' palettes. Retrieved from https://CRAN.R-project.org/package=rcartocolor

Pinheiro, J., Bates, D., DebRoy, S., Sarkar, D., \& R Core Team. (2017). nlme: Linear and nonlinear mixed effects models. Retrieved from https://CRAN.R-project.org/package $=$ nlme

Plate, T., \& Heiberger, R. (2016). Abind: Combine multidimensional arrays. Retrieved from https://CRAN.R-project.org/package=abind

Plummer, M., Best, N., Cowles, K., \& Vines, K. (2006). CODA: Convergence diagnosis and output analysis for mcmc. $R$ News, 6(1), 7-11. Retrieved from https://journal.r-project.org/archive/

R Core Team. (2017). R: A language and environment for statistical computing. Vienna, Austria: R Foundation for Statistical Computing. Retrieved from https://www.R-project.org/

Revelle, W. (2017). Psych: Procedures for psychological, psychometric, and personality research. Evanston, Illinois: Northwestern University. Retrieved from https://CRAN.R-project.org/package=psych

Robinson, D. (2017). Broom: Convert statistical analysis objects into tidy data frames. Retrieved from https://CRAN.R-project.org/package=broom

Saffran, J. R., Johnson, E. K., Aslin, R. N., \& Newport, E. L. (1999). Statistical learning of tone sequences by human infants and adults. Cognition, 70(1), 27-52. doi:10.1016/S0010-0277(98)00075-4

Solt, F., \& Hu, Y. (2015). interplot: Plot the effects of variables in interaction terms. Available at The Comprehensive R Archive Network (CRAN). Retrieved from 
https://CRAN.R-project.org/package=interplot

Venables, W. N., \& Ripley, B. D. (2002). Modern applied statistics with s (Fourth.). New York: Springer. Retrieved from http://www.stats.ox.ac.uk/pub/MASS4

Vigliocco, G., Meteyard, L., Andrews, M., \& Kousta, S. (2009). Toward a theory of semantic representation. Language and Cognition, 1(2), 219-247.

doi:10.1515/LANGCOG.2009.011

Wickham, H. (2009). Ggplot2: Elegant graphics for data analysis. Springer-Verlag New York. Retrieved from http://ggplot2.org

Xie, Y. (2015). Dynamic documents with $R$ and knitr (2nd ed.). Boca Raton, Florida: Chapman; Hall/CRC. Retrieved from https://yihui.name/knitr/

Xie, Y. (2016). Bookdown: Authoring books and technical documents with $R$ markdown. Boca Raton, Florida: Chapman; Hall/CRC. Retrieved from https://github.com/rstudio/bookdown

Zwaan, R. A. (2004). The immersed experiences: toward an embodied theory of language comprehension. In B. H. Ross (Ed.), The psychology of learning and motivation (Vol. 44, pp. 35-62). New York, NY, US: Elseview Science. Retrieved from http://citeseerx.ist.psu.edu/viewdoc/download?doi=10.1.1.588.3040\{\\&\}rep=rep1\{\\&\}type=pdf 